MATHEMATICAL MODELLING OF

POPULATION DYNAMICS

BANACH CENTER PUBLICATIONS, VOLUME 63

INSTITUTE OF MATHEMATICS

POLISH ACADEMY OF SCIENCES

WARSZAWA 2004

\title{
A BEGINNER'S GUIDE TO ADAPTIVE DYNAMICS
}

\author{
ODO DIEKMANN \\ Mathematisch Instituut, Universiteit Utrecht \\ P.O. Box 80010, NL 3508 TA Utrecht, The Netherlands \\ E-mail: diekmann@math.uu.nl
}

\begin{abstract}
The aim of these notes is to illustrate, largely by way of examples, how standard ecological models can be put into an evolutionary perspective in order to gain insight in the role of natural selection in shaping life history characteristics. We limit ourselves to phenotypic evolution under clonal reproduction (that is, we simply ignore the importance of genes and sex). Another basic assumption is that mutation occurs on a time scale which is long relative to the time scale of convergence to an ecological attractor.

We begin by illustrating the idea of interaction via environmental variables through the example of competition for substrate in the chemostat. In this context we explain the trait/strategy substitution sequence, capturing how successful invaders/mutants outcompete the resident and then become the new resident. We also introduce the PIP, the pairwise invasibility plot, as a convenient graphical tool to study the adaptive dynamics of a one-dimensional trait.

We high-light the pessimization principle: if the environmental condition is one-dimensional, mutation and natural selection inevitably lead to deterioration/Verelendung. We illuminate the Tragedy of the Commons as well as evolutionary suicide and, while we're about it, adaptive dynamics as an added feature to a bifurcation diagram (with, possibly, AD-induced branch switching).
\end{abstract}

Then we rewrite the invasion exponent as a function of resident and invader trait, define the selection gradient and turn to the core of the theory: the classification of singular points (where the selection gradient vanishes) in terms of ESS, CSS, mutual invasibility, converging - and diverging dimorphisms and branching points. An extensive collection of allied examples focuses on the timing of reproduction of semelparous organisms. We show how to analyse steady states of structured population models, how fitness measures relate to the dimension of the environmental condition and to the specific form of density dependence and we establish the central role of the ideal free distribution (i.e., the principle of indifference). We also describe the "resident strikes back" phenomenon. In the final section we very concisely sketch the wider perspective, alternative theories and the agenda of AD.

An almost identical earlier version of these notes appeared in: Summer School on Mathematical Biology, A. Margheri, C. Rebelo, F. Zanolin (eds.), CIM, Lisboa, Portugal, 2002.

2000 Mathematics Subject Classification: 92D15, 92D25. 
1. The ecological feedback loop. The starting point for a physiologically structured population model $[6,13,15,17,18,25,28,32,58,59,66,87]$ is a description of the life history of individuals, focusing in particular on everything related to

- maturation,

- survival,

- reproduction.

So one investigates how mechanisms at the $i$-level ( $i$ for individual) generate phenomena at the $p$-level ( $p$ for population). E.g., cannibalism may prevent extinction when adult food is scarce $[74,86,52]$ and variability in maturation delay, due to competition for food, may generate $p$-fluctuations with a certain period $[16,14]$.

Nonlinearity, i.e., dependence among individuals, is incorporated in a two-step procedure:

(i) Maturation, survival and reproduction may also depend on (relevant components of the) environmental conditions, which are "defined" by the requirement that the individuals are independent from each other whenever these are prescribed, say as a function of time. Examples are light intensity for plants, food concentration, predation pressure (i.e., the predation component to the force of mortality) and force of infection.

(ii) In turn, the environmental conditions are determined by the individuals constituting the population(s), e.g. by shading, consumption, predation, making contacts at which infective agents can be transmitted.

So interaction is not direct, but via environmental conditions. Or, in other words, the deterministic dynamics of well-mixed populations is governed by a feedback loop at the ecological time scale.

2. The evolutionary feedback loop. In $p$-models the $i$-life history is the key ingredient. But how has it itself evolved? Did natural selection shape it? Can we understand behavioural and physiological properties of individuals as the outcome of a process of adaptation at the much longer evolutionary time scale?

A popular, but naive, view is that survival of the fittest leads to optimal adaptation to the environment. In the preceding section we emphasized that the environment is (partly) determined by ecological interaction, so, in a sense, it co-evolves. And this phenomenon is at the heart of Adaptive Dynamics.

Unfortunately the fact, that the carrier of information in the process of inheritance, DNA, has been identified, doesn't make understanding natural selection simple. Selection acts on phenotypes and the map genotype $\rightarrow$ phenotype is, in almost all cases, extremely complicated and not understood at all. Moreover, sexual reproduction involves mating, a possibly rather subtle process. But even if we restrict our attention to random mating the map genotype $\times$ genotype $\rightarrow$ genotype is awfully more complicated than a simple Mendel picture suggests, due to the subtleties of crossing over/recombination when several loci are involved $[2,5,11,34,88]$.

So approximations are called for (like when you do solid state physics and ignore that, in principle, everything should follow from understanding elementary particles). In the 
framework of Quantitative Genetics these are based on things like many loci, additive effects and Gaussian distributions. The alternative is to ignore genes and sex all together and study phenotypic evolution. In other words, pretend that reproduction is clonal. Variability is then generated by mutations, which are usually assumed to be rare (as well as small).

The theory of phenotypic evolution comes in two brands. The earliest is based on direct interaction and game theory, with evolutionary success being measured in terms of (an often rather mysterious) pay off [63]. The second, Adaptive Dynamics, is only about 15 years old. It focuses on the effect of the ecological feedback loop and evolutionary success hinges on the positivity of the long term $p$-growth rate of a mutant in the environmental conditions set by the current resident.

\section{Adaptive Dynamics step-by-step}

Step 1. Single out a trait (or, in alternative denomination: a strategy repertoire), a particular aspect of phenotype (e.g., the fraction of ingested energy which is scheduled to reproduction). Here we shall restrict our attention to one-dimensional traits, meaning those that can be represented by one real number.

Step 2. Imagine a monomorphic (for this particular trait) population, called the resident (the word "resident" is used both to denominate the population, the type of individual and the trait value, depending on the context). Do invasibility analysis. That is, imagine a mutant with a (slightly) different trait and find out whether it starts a growing population (in the environmental conditions as set by the resident). This is a linear test and therefore it is relatively easily carried out. In principle it is based on a deterministic description of $p$-growth, but one can incorporate a branching process correction for the possibility of extinction of the mutant even when it has the potential to grow.

Note: next to "invasibility" one sees also "invadibility", "invadability" and "invasability" and this author has never understood whether there is any difference in meaning; could this be a case of neutral evolution?

Step 3. Investigate what will be the outcome of the competition between the resident and a successful invader. Will the invader outcompete the resident and thus become the new resident? Or will they coexist indefinitely (on the ecological time scale) and thus constitute a dimorphic resident population? The answer to such questions requires a nonlinear analysis. Unfortunately there are no generally applicable tools available and only in very special cases can answers be rigorously justified. Yet there are certain patterns that give confidence in conjectures for far more general cases $[43,56]$.

Step 4. On the basis of the information gained in steps 2 and 3, try to determine which traits can enter the stage as successful mutants and, once on stage, are invulnerable in the sense that no subsequent mutant can drive them to extinction. The key notion here is that of unbeatable strategy [49] or ESS for Evolutionarily Stable Strategy [62] which is a trait such that, when the vast majority of the individuals has it, no rare mutant with a different trait can increase in numbers. However, as we shall explain below, this property in effect characterizes a (monomorphic) Steady Strategy for the Adaptive 
Dynamics which may be a repellor rather than an attractor, so the "Stable" is a bit of a misnomer. The following two intuitive (rather than formal) examples underline that an unbeatable strategy has, per se, nothing to do with optimality:

(i) In the UK driving to the left is unbeatable, yet in the rest of Europe driving to the right is unbeatable. In other words, when there is no intrinsic advantage to any strategy, it may be that the majority sets the rules.

(ii) From a biological point of view most males are a waste (just think about a harem!). Yet a sex ratio of $\frac{1}{2}$ is very common. Fisher [40,9] gave the following explanation: every child has both a father and a mother, so if the sex ratio is female biased, males get more offspring than females. So if, in that situation, a mutant mother shifts her sex ratio towards sons, she gets more grandchildren than the average female. Note that in this example an intrinsically better trait is "beatable" because of a minority advantage.

4. Competition for food in the chemostat: a warming-up. The chemostat ([80], see Figure 1) is a laboratory device to culture micro-organisms, like algae or bacteria. In a vessel of volume $V=1$ all they need to grow is provided in excess, except for one substance (e.g., phosphate) which accordingly is called the limiting substrate or resource. Its concentration is denoted by $S$. Fresh medium containing substrate with concentration $S_{0}$ is pumped into the vessel at rate $D$. At the same rate $D$ fluid is pumped out, containing substrate with concentration $S$ (since the chemostat is continuously stirred) and microorganisms with concentration $X$.

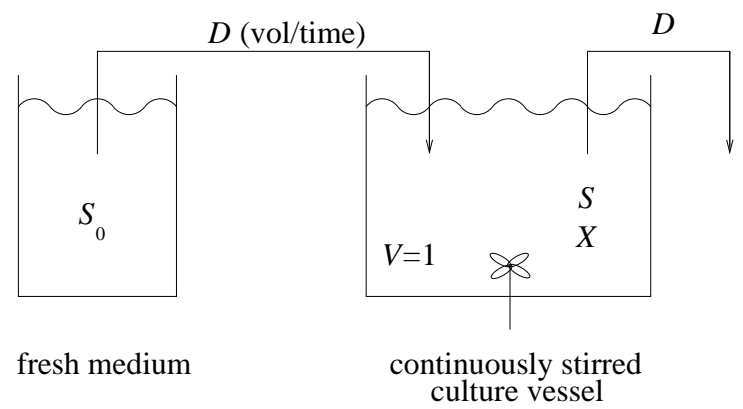

Fig. 1. The chemostat

Each micro-organism consumes substrate at a rate $g$ which depends on the availability $S$. Quite generally, $g$ has the form depicted in Figure 2 (linear for low $S$, increasing, saturating for large $S$; just as the output of a factory is proportional to the rate at which raw material is provided when this rate is low, but is determined by the capacity of the factory, not by the input, when this rate is high). Various submodels for the short-time-scale consumption process such as the Holling time budget model (explained in, e.g., [66] or the Michaelis-Menten enzyme reaction model (presented in, e.g., [79]) yield expressions for $g$ of the form

$$
g(S)=\frac{a S}{1+a h S}
$$




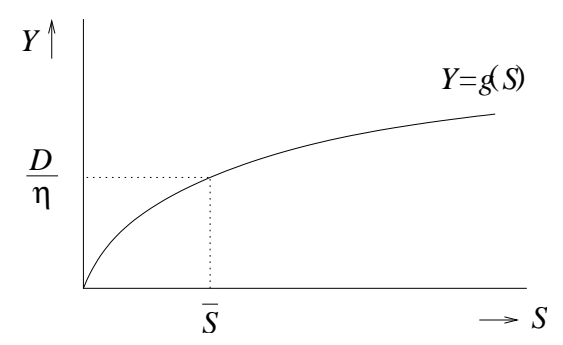

Fig. 2. Graphical representation of the rate $g$ at which a micro-organism consumes substrate. The population of micro-organisms will steer $S$ to $\bar{S}$ provided $\bar{S}<S_{0}$.

which are called a Monod, Michaelis-Menten or Holling type 2 function.

As an idealized description of reality we assume that the consumed substrate is immediately transformed into new micro-organisms, with conversion efficiency $\eta$ (when $X$ represents biomass/volume, this is not that strange an assumption!). The system of ordinary differential equations (beware that the volume $\mathrm{V}$ of the vessel doesn't appear since we choose the unit of volume such that $\mathrm{V}=1$ )

$$
\begin{array}{ll}
\frac{d S}{d t}=D S_{0} & -D S-g(S) X, \\
\frac{d X}{d t}= & -D X+\eta g(S) X,
\end{array}
$$

reflects all these assumptions and tells us how $S$ and $X$ will change in the course of time by inflow, outflow and consumption/division. From the point of view of the micro-organism, the environmental condition is completely described by the substrate concentration $S$. Our first task is to investigate the dynamical behaviour generated by (4.2).

Observation 1: The $p$-attractor is a steady state.

Argument: Since $\frac{d}{d t}\left[S+\frac{1}{\eta} X\right]=D\left\{S_{0}-\left[S+\frac{1}{\eta} X\right]\right\}$ necessarily

$$
S(t)+\frac{1}{\eta} X(t) \rightarrow S_{0} \quad \text { for } t \rightarrow \infty .
$$

So the large time behaviour is governed by a one-dimensional problem and, therefore, convergence to a limit must occur.

Observation 2: $S$ settles at $\bar{S}$, the level at which the $p$-growth rate of the microorganism equals zero, formally defined by the equation

$$
g(\bar{S})=\frac{D}{\eta}
$$

provided $\bar{S}<S_{0}$.

Argument: The introduction of the micro-organism in an "empty" chemostat (i.e., when $\left.(S, X)=\left(S_{0}, 0\right)\right)$ leads to $p$-growth iff $-D+\eta g\left(S_{0}\right)>0$ which, given the monotonicity of $g$, translates into $\bar{S}<S_{0}$ (see Figure 2). A steady-state with $X \neq 0$ should have $S=\bar{S}$. The corresponding value for $X$ is found by solving $D S_{0}-D \bar{S}-g(\bar{S}) X=0$ and so it is positive iff $\bar{S}<S_{0}$. 
Now consider the conversion efficiency $\eta$ as the trait of the micro-organism (please stop a moment to test your biological intuition on a very simple question answered below: how will $\eta$ evolve by the combined action of mutation and natural selection?). When there is a monomorphic resident with trait $\eta_{\text {res }}$, the environmental condition is, according to the observations above, set at $\bar{S}_{\eta_{\text {res }}}$ (the quantity $\bar{S}$ depends on $\eta$ and there is now a need to express this explicitly in the notation). A mutant with trait $\eta_{\text {inv }}$ will start a growing population when

$$
\eta_{\text {inv }} g\left(\bar{S}_{\eta_{\text {res }}}\right)>D
$$

(the deterministic certainty of this statement needs a stochastic correction, as either the founder or all of its clan may be washed out before the clan is large enough for total wash-out to become unlikely). If, on the other hand

$$
\eta_{\text {inv }} g\left(\bar{S}_{\eta_{\text {res }}}\right)<D
$$

the clan is doomed to be washed out, i.e., to go extinct. We call the $p$-growth rate

$$
\eta_{\text {inv }} g\left(\bar{S}_{\eta_{\text {res }}}\right)-D
$$

the invasion exponent and, also, the fitness of the mutant in the environmental condition set by the resident [67]. Since, by definition,

$$
\eta_{\text {res }} g\left(\bar{S}_{\eta_{\text {res }}}\right)-D=0
$$

we find that an invader is successful iff its trait-value is higher than that of the resident. So the outcome of the linear invasibility test is that $\eta$ will increase by natural selection (as you had doubtlessly predicted).

Is the nonlinear competition such that a successful invader drives the resident to extinction? In other words, does a successful invader become the new exclusive ruler of the mini-world that the chemostat constitutes? The fact that mutual invasibility does not occur (indeed, if $\eta_{1}>\eta_{2}$ then type 1 individuals can invade in the environmental conditions as set by a type 2 resident, but not vice versa) indicates that the answer is "yes". To decide whether it is "yes" indeed, we study the full nonlinear competition system

$$
\begin{array}{lll}
\frac{d S}{d t}=D S_{0} & -D S-g(S) X & -g(S) Y, \\
\frac{d X}{d t}= & -D X+\eta_{\mathrm{res}} g(S) X, & \\
\frac{d Y}{d t}= & -D Y & +\eta_{\mathrm{inv}} g(S) Y .
\end{array}
$$

Since $S(t)+\frac{1}{\eta_{\mathrm{res}}} X(t)+\frac{1}{\eta_{\mathrm{inv}}} Y(t) \rightarrow S_{0}$ for $t \rightarrow \infty$ we can restrict our attention to the 2-dimensional problem for $X$ and $Y$ with, in the differential equations $S$ put equal to $S_{0}-\frac{1}{\eta_{\mathrm{res}}} X-\frac{1}{\eta_{\mathrm{inv}}} Y$ (see $[80,82]$ for careful justification).

The local invasibility analysis based on linearization yields the information depicted in Figure 3. An internal (to the positive quadrant) steady state does not exist (indeed, it would require that one quantity, $\bar{S}$, is such that both the $p$-growth rate $-D+\eta_{\text {res }} g(\bar{S})$ of $X$ and the $p$-growth rate $-D+\eta_{\text {inv }} g(\bar{S})$ of $Y$ are zero, which clearly is impossible if $\left.\eta_{\text {inv }} \neq \eta_{\text {res }}\right)$. The Poincaré-Bendixson theory of planar dynamical systems then implies 


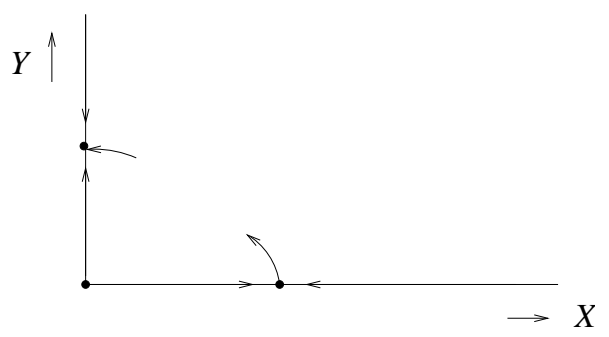

Fig. 3. Graphical representation of (non)invasibility

that all orbits in the interior of the positive quadrant are attracted towards the stable steady state at the $Y$-axis (to make the argument precise, one needs to check that all orbits remain bounded; this is straightforward).

The conclusion is that a successful invasion generates a trait substitution: after a period of competitive interaction the population becomes monomorphic again, but the trait value of the resident has changed. Now assume time scale separation, in particular that the period of competitive interaction is short relative to the long evolutionary time scale determined by the mutation process. Then we can think of the trait substitution as occurring more or less instantaneously. So then we may describe the changes in the trait by the combined influence of mutation and natural selection as a trait substitution sequence.

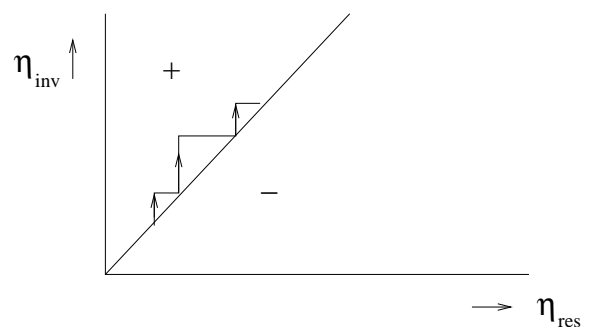

Fig. 4. Pairwise Invasibility Plot for unconstrained evolution of conversion efficiency

The information concerning the adaptive dynamics of the trait $\eta$ can be conveniently represented graphically in a so-called PIP, a Pairwise Invasibility Plot. See Figure 4. At the $45^{\circ}$ line $\eta_{\text {inv }}=\eta_{\text {res }}$ there is, of course, neutrality. This line separates the + region of $\left(\eta_{\text {res }}, \eta_{\text {inv }}\right)$-pairs with a successful invader from the - region of pairs such that the invader fails. It is convenient to think of a monomorphic state for the adaptive dynamical system as a point on the $45^{\circ}$ line. A mutation then yields a jump to a point on the vertical line with fixed $\eta_{\text {res }}$. When this point lies in the - region, we simply jump back to the starting point so that, essentially, nothing has happened. Therefore we may as well (and will indeed) simply ignore unsuccessful mutations. When, on the other hand, the mutation yields a point in the + region, we need to do the nonlinear competition analysis. In the present case we found that the successful invader takes over completely. This "taking over" is represented by a horizontal move to the $45^{\circ}$ line. Repeating the trait 
substitution again and again we generate a stair climbing evolution. The heights of the steps are determined by the random mutation process, but the fact that $\eta$ increases, rather than decreases, is due to natural selection (here we take advantage of the one-dimensional character of the trait: by the order structure of the real line, $\eta$ can only evolve in two directions, up or down; contrast this with the possibilities in $\mathbb{R}^{n}$ with $n \geq 2$ ). It makes sense to think of the steps as being rather small.

Thus far we pretended that there were no physiological costs whatsoever associated with an increase of $\eta$. Next, let us complicate the matter by postulating a trade-off between the conversion efficiency $\eta$ and the up-take efficiency $h^{-1}$ featuring in the expression (4.1) for $g$ (note that $\lim _{S \rightarrow \infty} g(S)=h^{-1}$ so that $h^{-1}$ equals the maximal up-take rate). The nature of the trade-off is presented in Figure 5 and its caption.

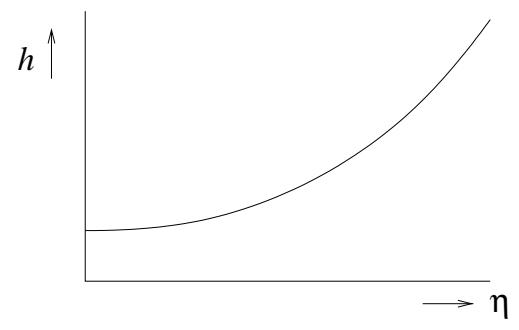

Fig. 5. Graphical representation of the way $h$ increases when $\eta$ increases, corresponding to a limited biochemical machinery, which can be either used for improving conversion or for improving up-take, but not for both at the same time

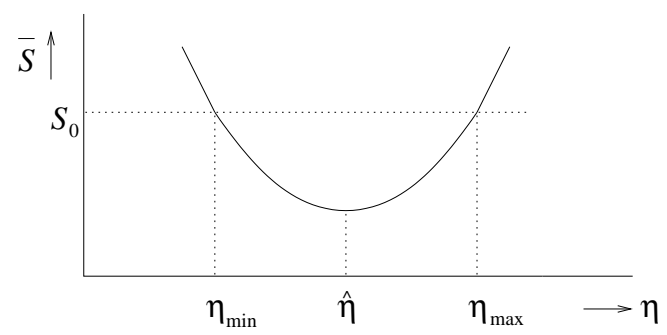

Fig. 6. Only $\eta$-values in the interval $\left(\eta_{\min }, \eta_{\max }\right)$ yield a viable population

When $g$ is given by (4.1) we have explicitly

$$
\bar{S}=\frac{1}{a\left(\frac{\eta}{D}-h(\eta)\right)}
$$

The graphical presentation in Figure 6 of the relation (4.5), with $h$ as depicted in Figure 5, tells us two things:

- the set of viable $\eta$ values is a finite interval,

- with the exception of $\hat{\eta}$, the $\eta$-value for which $\bar{S}$ achieves its minimum, each $\eta$-value is paired to exactly one other $\eta$-value characterized by equality of the corresponding $\bar{S}$ value. 
The second point implies that in the PIP there is, apart from the $45^{\circ}$ line, a curve of neutrality. This curve connects $\left(\eta_{\text {res }}, \eta_{\text {inv }}\right)=\left(\eta_{\min }, \eta_{\max }\right)$ to $\left(\eta_{\text {res }}, \eta_{\text {inv }}\right)=\left(\eta_{\text {max }}, \eta_{\text {min }}\right)$, intersects the $45^{\circ}$ line at $\hat{\eta}$, the $\eta$-value at which $\bar{S}$ is minimal, is symmetric with respect to reflection in the $45^{\circ}$ line, i.e., with respect to interchanging $\eta_{\text {res }}$ and $\eta_{\text {inv }}$, and so has a tangent line with slope -1 at the intersection with the $45^{\circ}$ line. Apart form the + and

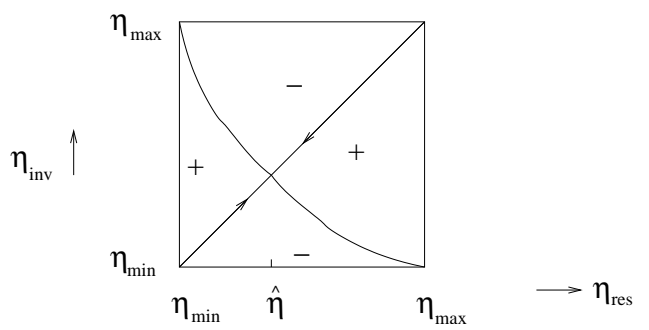

Fig. 7. PIP in the case of $\eta-h$ trade-off

- signs and the arrows along the $45^{\circ}$ line, the features of the PIP in Figure 7 are just a graphical presentation of these observations. It remains to justify the pattern of + and - signs (the arrows just indicate that, given the sign pattern, the adaptive dynamics of $\eta$ are such that $\eta$ increases when smaller than $\hat{\eta}$ and decreases when larger than $\hat{\eta}$ ). To do so we observe that

(i) $S \mapsto \frac{a S}{1+a h S}$ is monotone increasing,

(ii) $\eta \frac{a \bar{S}_{\eta}}{1+a h(\eta) \bar{S}_{\eta}}=D$ so, in particular, independent of $\eta$,

(iii) the invasion exponent is $\eta_{\mathrm{inv}} \frac{a \bar{S}_{\eta_{\mathrm{res}}}}{1+a h\left(\eta_{\mathrm{inv}}\right) \bar{S}_{\eta_{\mathrm{res}}}}-D$,

and from these observations conclude that $\bar{S}_{\eta_{\mathrm{inv}}}<\bar{S}_{\eta_{\mathrm{res}}}$ implies success for the invader, while the opposite inequality implies failure. On the other hand, we deduce from Figure 6 that $\bar{S}_{\eta_{\mathrm{inv}}}<\bar{S}_{\eta_{\mathrm{res}}}$ for $\eta_{\mathrm{inv}}$ in between $\eta_{\mathrm{res}}$ and the point that is paired to $\eta_{\text {res }}$. Hence the + should be above the $45^{\circ}$ line when $\eta_{\text {res }}$ is less than the value $\hat{\eta}$ at which $\bar{S}$ achieves its minimum, and below the $45^{\circ}$ line when $\eta_{\text {res }}$ exceeds $\hat{\eta}$. We now read off from the PIP depicted in Figure 7 that:

(i) $\hat{\eta}$ is a global ESS since the vertical line through $\hat{\eta}$ is entirely in the - region,

(ii) the trait substitution sequence converges to $\hat{\eta}$.

Apparently the coin of optimal adaptation to the environment has another side to it: by the ecological feedback through consumption, the environment becomes ever more harsh, i.e., $\bar{S}_{\eta}$ decreases to its minimum along the trait substitution sequence. This is sometimes called a pessimization principle: mutation and natural selection lead to a deterioration of the environmental condition, a Verelendung. We end up with the worst of all possible environments. Reassuringly a crucial aspect is that in this example the environmental condition is described by just one number, the steady state value of $S$ (again it is the order structure of $\mathbb{R}$ that limits the possibilities rather severely). 
5. The general features of pessimization. Suppose that a population of individuals with trait $x$ grows under constant environmental conditions, characterized by a variable $I$, like

$$
e^{r(x, I) t}
$$

(in Section 4 we had the correspondence $x \sim \eta, I \sim S$ and

$$
\left.r(x, I)=x \frac{a I}{1+a h(x) I}-D\right) .
$$

Now assume that both $x$ and $I$ are scalars, i.e., they are fully specified by one real number. Also assume that $r$ is monotone in $I$, say strictly increasing. The steady state equation $r(x, I)=0$ then has a unique (for given $x$ ) solution $I=\bar{I}(x)$. Assume that the population dynamics (which we do not describe in detail, as only the growth rate $r$ figures in the rest of the argument) is such that the steady state is an attractor (or, even, a global attractor; as a side-remark we mention that very often such assumptions are not verified even when the population dynamics is actually specified in detail). The monotonicity in $I$ and the identity $r(x, \bar{I}(x))=0$ imply that

$$
\operatorname{sign} r(y, \bar{I}(x))=\operatorname{sign}(\bar{I}(x)-\bar{I}(y)) .
$$

So the linear invasibility test for a mutant with trait $y$ in the environmental conditions set by a resident with trait $x$

- predicts success if $\bar{I}(y)<\bar{I}(x)$,

- guarantees failure if $\bar{I}(y)>\bar{I}(x)$.

Now assume that successful invasion results in take-over, i.e., leads to the extinction of the resident. Then the adaptive dynamics is described by a trait substitution sequence and at every substitution the value of $\bar{I}$ necessarily decreases. Accordingly the sequence leads to a trait $\hat{x}$ at which $x \mapsto \bar{I}(x)$ has a (local) minimum. Such a trait $\hat{x}$ is automatically a (local) ESS. See [70, 69, 29].

6. A manifestation of the Tragedy of the Commons. The example of Section 4 warns us against naive optimistic views about natural selection. The example of this section (which was concocted by Kalle Parvinen, in response to provocative remarks by the author) is even stronger in this respect: it shows how trait substitution may drive a population to extinction. This phenomenon is called "evolutionary suicide" [38, 46, 48].

But the example has as an additional aim to demonstrate how adaptive dynamics may be visualized by adding "arrows" to a bifurcation diagram.

Again we consider a resource-consumer model but this time the resource is selfreproducing too. Moreover, an Allee effect is incorporated in the resource dynamics, meaning that the resource is bound to go extinct once its density/concentration drops below a certain threshold. The population dynamical system is generated by the differential equations

$$
\begin{aligned}
& \dot{S}=S\left(\frac{a S}{1+S}-b-c S\right)-\theta S X \\
& \dot{X}=X(\eta \theta S-\mu) .
\end{aligned}
$$


We consider the parameter $\theta$ as describing a trait of the consumer. Clearly mutants with larger $\theta$ will invade successfully in the environmental conditions set by a resident with smaller $\theta$ (no matter whether the environmental condition, i.e., $S$, is constant or fluctuates; indeed, the per capita growth rate is a strictly increasing function of $\theta$, for every positive $S$ ). Moreover, the successful invader population will, for the same reason, outcomplete the resident, so trait substitution will occur. In conclusion, the adaptive dynamics of $\theta$ is strict increase.

It remains to investigate how the dynamics generated by (6.1) depends on $\theta$. We present the outcome of this investigation in the form of the bifurcation diagram in Figure 8.

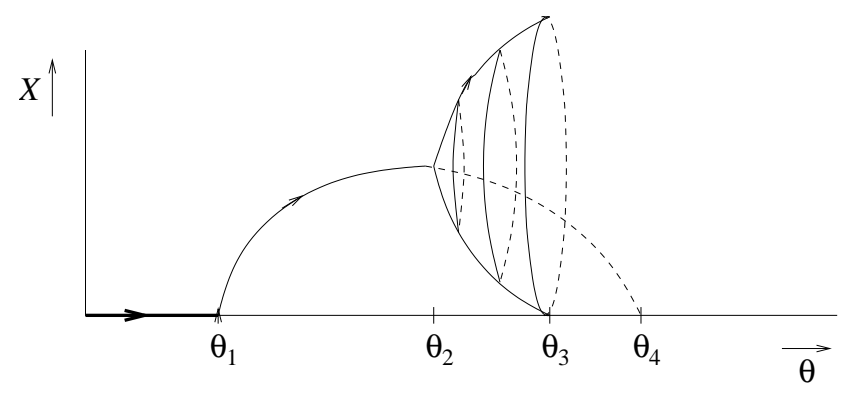

Fig. 8. Bifurcation diagram for (6.1). The adaptive dynamical increase of $\theta$ is indicated by the arrows (but, admittedly, the arrow between 0 and $\theta_{1}$ is meaningless).

To understand this diagram, it is helpful to draw the phase plane of $(6.1)$, and to note that the vertical consumer isocline $S=\mu / \eta \theta$ shifts to the left whereas the resource isocline $X=\frac{1}{\theta}\left(\frac{a S}{1+S}-b-c S\right)$ only loses height, but does not shift, when $\theta$ increases (here we presume that the $S$-axis is horizontal and the $X$-axis vertical, just as in Figure 9 ). For $0<\theta<\theta_{1}$ there is no internal steady state, the consumer goes extinct. For $\theta_{1}<$ $\theta<\theta_{2}$ there is a stable internal steady state. At $\theta=\theta_{2}$ the vertical consumer isocline intersects the resource isocline in the top. As $\theta$ passes $\theta_{2}$ a Hopf bifurcation occurs and for $\theta>\theta_{2}$ there is at first a stable limit cycle surrounding the now unstable internal steady state.

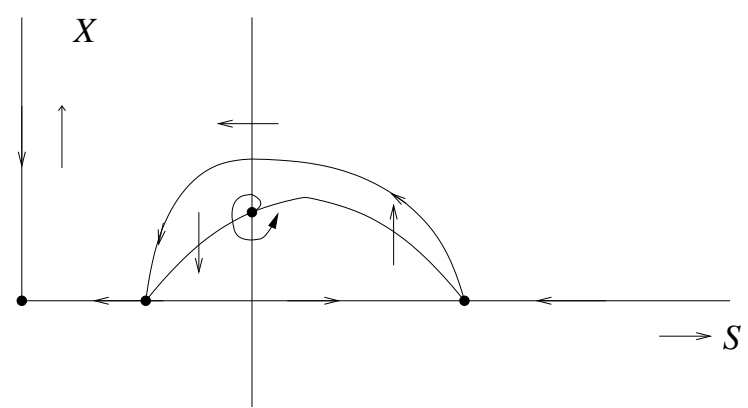

Fig. 9. Phase plane of (6.1) for $\theta=\theta_{3}$ at which there is a heteroclinic connection between the two saddle-points at the $S$-axis 
As $\theta$ increases this limit cycle "grows" in amplitude, but much stronger in period, until, for a critical parameter value called $\theta_{3}$, it "dies" in a heteroclinic cycle formed by the piece of the $S$-axis in between the two saddle points and an internal orbit that connects the upper saddle point to the lower one. For $\theta>\theta_{3}$ the unstable manifold of the upper saddle point "ends" in the origin (which is actually a stable steady state for all $\theta$ ). With the stable manifold of the lower saddle point as the only exception, all orbits in the interior "end" in the origin. In other words, both the resource and the consumer go extinct for $\theta>\theta_{3}$.

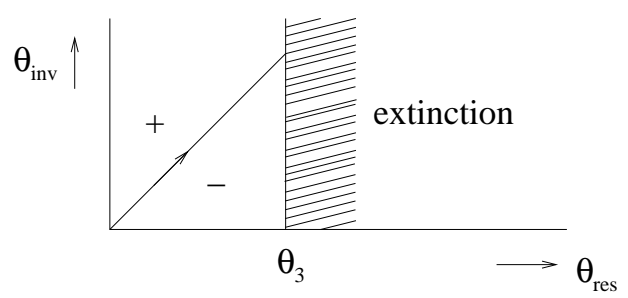

Fig. 10. PIP for (6.1) with consumer trait $\theta$

The concomitant evolutionary suicide is depicted in both Figure 8 and Figure 10. Connoisseurs will recognize the Tragedy of the Commons, which derives from the advantage of being rare: you profit, but don't pay the price (for a while, that is, not until you are not rare any more; so it is a short term policy only).

One might indeed say that examples of this kind illustrate the need for having the Law, as well as the police to enforce it.

In conclusion of this section we mention the work of Dercole, Ferrière \& Rinaldi [20], in which they find evolutionary cycling involving attractor switching by rigging a bifurcation diagram as in Figure 11 with arrows indicating the direction of adaptive parameter change.

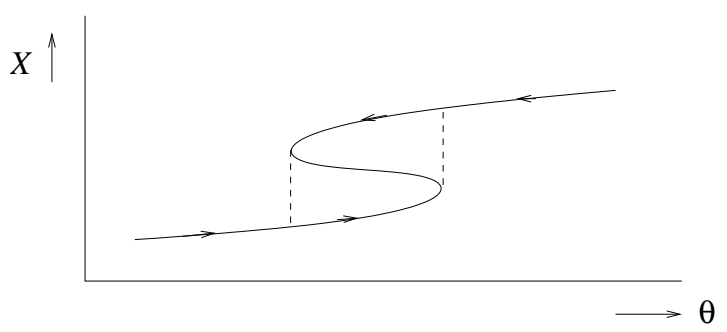

Fig. 11. Evolutionary cycling involving attractor switching, as found in [20]

EXERCISE 6.1. Cannibalism is both more common than most people are aware and less prominent than an evolutionary biologist might expect at first $[12,19,28,52,74,86]$. Here, to sharpen our intuition, we consider a very very caricatural model.

The equation

$$
\frac{d x}{d t}=r x-k x^{2}+(1-\epsilon) k x^{2}
$$


describes a population which, in the absence of cannibalism, would grow exponentially with rate $r$. The negative efect of cannibalism, the additional death rate, is incorporated in the term $-k x^{2}$. The positive effect, the production of offspring from energy obtained by intraspecific predation, is represented by the term $(1-\epsilon) k x^{2}$. We assume $0<\epsilon<1$, so there is a net loss. Accordingly cannibalism acts as a negative feedback mechanism that regulates the population size. The parameter $k$ describes the degree of cannibalistic activity. It will be the trait under consideration.

(i) Check that (6.2) has the steady state $\bar{x}=\frac{r}{\epsilon k}$ as a global attractor.

(ii) Explain the rationale underlying the equation

$$
\frac{d y}{d t}=r y-k_{\mathrm{res}} \bar{x} y+(1-\epsilon) k_{\mathrm{inv}} \bar{x} y
$$

for the initial dynamics of an invader population.

(iii) Show that the invasion exponent equals

$$
r \frac{(1-\epsilon)}{\epsilon}\left(\frac{k_{\text {inv }}}{k_{\text {res }}}-1\right)
$$

and conclude that the invader will be successful if and only if $k_{\mathrm{inv}}>k_{\text {res }}$.

(iv) Derive the system

$$
\begin{aligned}
& \frac{d x}{d t}=r x-k_{1} x^{2}-k_{2} y x+(1-\epsilon) k_{1}(x+y) x, \\
& \frac{d y}{d t}=r y-k_{1} x y-k_{2} y^{2}+(1-\epsilon) k_{2}(x+y) y,
\end{aligned}
$$

for the nonlinear interaction. Check that this is a Voltera-Lotka competition system with, in case $k_{2}>k_{1}$, the boundary steady state $(x, y)=\left(0, \frac{r}{\epsilon k_{2}}\right)$ as the global attractor. Conclude that successful invasion results in trait substitution.

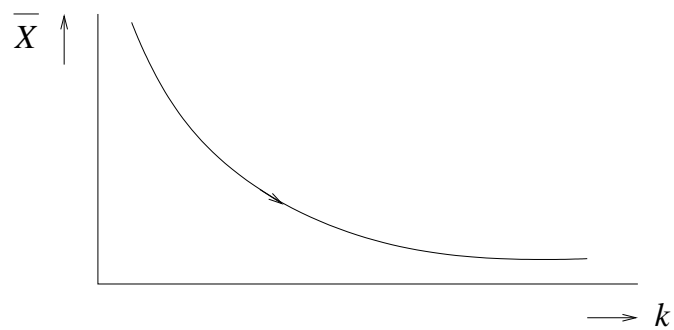

Fig. 12. Adaptive dynamics of cannibalistic activity and its impact on population size

(v) Relate the results (which are depicted in Figure 12) to:

(a) the Tragedy of the Commons,

(b) the Pessimization Principle of Section 5 (what quantity does correspond to I?),

(c) evolutionary suicide.

(vi) Why should we doubt the conclusion that cannibalism will increase in strength for ever? Scrutinize the assumptions! Hints: (a) How would spatial structure affect the probability to cannibalize your own offspring rather than that of a random conspecific? (b) How would the ability to catch regular prey be affected if you keep hunting your own kind? 
7. The classification of singular points in PIP's. We now interrupt the stream of examples to present the main result of the theory developed so far. Here we are concerned with a one-dimensional trait $x$ undergoing small mutations on a long (relative to ecological dynamics) time scale.

Above we have advocated the use of variables characterizing relevant aspects of the environmental conditions as a key step in describing interaction. From the point of view of modeling and interpretation this is indeed a good idea. But in this section we shall suppress $I$ in the notation and write the invasion exponent as $s_{x}(y)$, where $x$ is the trait of the resident and $y$ the trait of the invader. This relates directly to PIP's and it is efficient: the essential structure is exposed first and detailed calculations for specific models are postponed. The notation presupposes that the ecological attractor is unique or, in case it is not, that we have additional information about the attractor concerned (recall Figure 11 and see [43]).

The formal definition reads: the invasion exponent $s_{x}(y)$ is the long term population growth rate of a mutant population with trait $y$ under environmental conditions as set by the resident population with trait $x$ (these conditions can be steady, periodic or even chaotic; clearly this distinction has influence on the ease with which $s_{x}(y)$ is computed, but not on the classification!)

We note right away the two useful identities

$$
\begin{gathered}
\frac{\partial s}{\partial x}+\frac{\partial s}{\partial y}=0 \\
\frac{\partial^{2} s}{\partial x^{2}}+2 \frac{\partial^{2} s}{\partial x \partial y}+\frac{\partial^{2} s}{\partial y^{2}}=0
\end{gathered}
$$

which hold along the diagonal $x=y$ and are straightforward consequences of the fundamental identity

$$
s_{x}(x)=0
$$

which itself is a direct corollary of the definition of $s$.

The sign of the selection gradient

$$
\left[\frac{\partial}{\partial y} s_{x}(y)\right]_{y=x}
$$

tells us in which direction the trait will evolve from the current resident strategy $x$; if the sign is positive the trait increases and if it is negative the trait decreases (here we take for granted that successful invasion implies trait substitution; see [43, 56] for conditions that yield a rigorous justification). A singular point $\bar{x}$ is a trait value at which the selection gradient vanishes, so where the first order test fails to tell us which way natural selection will lead us. If $s$ is smooth then, because of (7.3), it has the form $s_{x}(y)=(x-y) \Phi(x, y)$ and the selection gradient equals $-\Phi(x, x)$. Solving $\Phi(x, y)=0$ in the neighbourhood of the known solution $(x, y)=(\bar{x}, \bar{x})$ we find, generically, a second curve on which $s_{x}(y)$ changes sign (the first being the $45^{\circ}$ line $y=x$ ). Conversely, singular points are geometrically found as intersections of such curves with the $45^{\circ}$ line. Now, have another look at Figure 7. 
We shall classify singular points on the basis of properties which are relevant for the adaptive dynamics in their neighbourhood. We shall introduce these properties via questions concerning the adaptive dynamics. Given a singular point $\bar{x}$, we consider the following four questions:

1. If the resident has strategy $\bar{x}$, can invaders with nearby strategies be successful? If the answer is "no", we call $\bar{x}$ a (local) ESS. As already explained in Section 3, an ESS is simply a steady strategy, i.e., a steady state for the adaptive dynamics.

2 . If the resident has a strategy $x$ near $\bar{x}$, will successful invaders be nearer to $\bar{x}$ ? If the answer is "yes", we call $\bar{x}$ a Convergence Stable Strategy (be careful: the abbreviation CSS is used to denote a Continuously Stable Strategy, which is by definition a Convergence Stable ESS; both names are awful, and comprehensible only in historical perspective: the adjective "stable" was already (but unjustly!) incorporated in ESS). A Convergence Stable Strategy is simply a (local) attractor for the adaptive dynamics, so could be called an EAS for Evolutionarily Attracting Strategy.

3. Can $\bar{x}$, as a mutant, invade successfully when a nearby strategy is the resident? In other words, can $\bar{x}$ be established in one go? (This question is not as relevant as the other three, as it concerns a bit of a detail. By incorporating the question nevertheless in the classification, the final result (see Figure 14) has a nice symmetric appearance. It is probably fair to say that this appearance served as the main motivation to include the question.)

4. Are there pairs of strategies $x, y$ near $\bar{x}$ such that both $s_{x}(y)>0$ and $s_{y}(x)>0$ ? If the answer is "yes", we say that mutual invasibility occurs. The consequence of mutual invasibility is that a (so-called protected) dimorphism may arise, rather than that a trait substitution happens. So we are led outside the monomorphic framework and we have to extend the formalism. We return to this important point below.

A standard pattern in mathematics is that a classification involves derivatives of one order higher than those underlying the definition of the objects to be classified (e.g., think of zeros, extrema, bifurcation points; of course in special, degenerate, cases one needs to compute still higher order derivatives to decide how an object fits in the classification). Here we shall encounter exactly that pattern: the operational classification of singular points is based on second order derivatives of $s$. The identity (7.2) tells us that two real numbers fully specify the three second order derivatives. As such we choose

$$
c_{11}=\frac{\partial^{2} s}{\partial x^{2}} \quad \text { and } \quad c_{22}=\frac{\partial^{2} s}{\partial y^{2}}
$$

(using, whenever needed, that $c_{12}=c_{21}=-\frac{1}{2}\left(c_{11}+c_{22}\right)$ where, of course, $c_{12}=\frac{\partial^{2} s}{\partial x \partial y}$ and $c_{21}=\frac{\partial^{2} s}{\partial y \partial x}$ ). Our aim is to divide the $\left(c_{11}, c_{22}\right)$-plane into regions according to the type of a singular point, where "type" refers to the answers to the four questions listed above.

It is always helpful if a geometric image can be associated with algebraic criteria. Here the right image involves the angle that the tangent line to the second curve, along which $s_{x}(y)$ vanishes, makes with the $45^{\circ}$ line and, additionally, the +- sign pattern in the four quadrants formed by the $45^{\circ}$ line and the second curve. So in Figure 14 each region 
in the $\left(c_{11}, c_{22}\right)$-plane is equipped with a representative picture of the relevant structure: the $45^{\circ}$ line, the tangent line and the sign pattern (in fact in Figure 14 the mirror image of this structure under reflection in the $45^{\circ}$ line is presented as well, to facilitate reaching conclusions concerning dimorphic dynamics). The fact that the classification involves only visually recognizable properties is very helpful, in particular if a PIP is constructed by way of numerical invasibility tests.

As it is traditional in PIP's (see Figures 4, 7 and 10), we shall put the resident strategy $x$ along a horizontal axis and the invader strategy $y$ along a vertical axis.

Question 1. In order for $\bar{x}$ to be a (local) ESS, the vertical line through $\bar{x}$ should lie (locally) in the region where $s<0$, so the function $y \mapsto s_{\bar{x}}(y)$ should have a (local) maximum in $y=\bar{x}$. The generic criterion for this to be the case is

$$
c_{22}<0
$$

(by "generic" we mean that we are aware of the fact that one could have, for instance, $c_{22}=0, c_{222}=0, c_{2222}<0$, but that we do not dwell upon such degenerate cases until the need arises in the context of a special example). For completeness we observe that if $c_{22}>0$ then $y \mapsto s_{\bar{x}}(y)$ has a (local) minimum in $y=\bar{x}$ and accordingly the trait can move both ways if $\bar{x}$ is the resident.

Question 2. In order for $\bar{x}$ to be Convergence Stable the selection gradient should be positive for $x<\bar{x}$ and negative for $x>\bar{x}$, so the derivative of the selection gradient should, generically, be negative. So the generic criterion for Convergence Stability is

$$
0>\left[\frac{\partial}{\partial x}\left(\left[\frac{\partial}{\partial y} s_{x}(y)\right]_{y=x}\right)\right]_{x=\bar{x}}=\left[\frac{\partial^{2} s}{\partial x \partial y}+\frac{\partial^{2} s}{\partial y^{2}}\right]_{y=x=\bar{x}}=c_{12}+c_{22}
$$

which, in view of (7.2), can be written as

$$
c_{11}>c_{22} \text {. }
$$

If the opposite inequality holds $\bar{x}$ is a repellor for the adaptive dynamics: if the resident has a strategy near $\bar{x}$, successful invaders will be further away from $\bar{x}$.

Question 3. In order for $\bar{x}$ to invade successfully, as a mutant, nearby strategies when these are resident, the horizontal line through $\bar{x}$ should lie (locally) in the region where $s>0$, so the function $x \mapsto s_{x}(\bar{x})$ should have a (local) minimum in $x=\bar{x}$. The generic criterion for this to be the case is

$$
c_{11}>0
$$

If the opposite inequality holds, it is still possible that $\bar{x}$ is CSS, i.e., both ESS and Convergence Stable, but ever smaller mutations are required to end up, asymptotically, in $\bar{x}$.

Question 4. Mutual invasibility requires that there are $x, y$ (arbitrarily) close to $\bar{x}$ such that both $s_{x}(y)>0$ and $s_{y}(x)>0$. Interchanging $x$ and $y$ corresponds geometrically to reflection in the $45^{\circ}$ line. Therefore, mutual invasibility requires that the line making an angle of $-45^{\circ}$ with the horizontal axis, so the line $y=2 \bar{x}-x$, lies (locally) in the region where $s>0$, i.e., the function $x \mapsto s_{x}(2 \bar{x}-x)$ should have a (local) minimum in 
$\bar{x}$. This function has first derivative $\frac{\partial s}{\partial x}-\frac{\partial s}{\partial y}$ and second derivative $\frac{\partial^{2} s}{\partial x^{2}}-2 \frac{\partial^{2} s}{\partial x \partial y}+\frac{\partial^{2} s}{\partial y^{2}}=$ $2\left(\frac{\partial^{2} s}{\partial x^{2}}+\frac{\partial^{2} s}{\partial y^{2}}\right)$. So the generic condition for mutual invasibility reads

$$
c_{11}>-c_{22} \text {. }
$$

It remains to investigate the consequences of mutual invasibility. If a successful mutant cannot outcompete the resident once the resident is becoming rare, we must get coexistence of two subpopulations with different traits. Or, in other words, the population becomes dimorphic. But what happens next? The invasion exponent $s_{x}(y)$ is now useless, as it presupposes a monomorphic resident. It has to be replaced by $s_{x_{1}, x_{2}}(y)$, where $x_{1}$ and $x_{2}$ specify the traits present in the resident population and $y$ still refers to a mutant (attentive readers will realize that this is not the end of the story and that a polymorphic resident population may consist of many more subpopulations characterized by a particular trait; see $[68,44,45]$ for lots of information on bookkeeping and consistency requirements). Clearly we should have $s_{x_{1}, x_{2}}\left(x_{1}\right)=0=s_{x_{1}, x_{2}}\left(x_{2}\right)$. Continuity considerations then suggest that $y \mapsto s_{x_{1}, x_{2}}(y)$ should have a maximum in between $x_{1}$ and $x_{2}$ if $y \mapsto s_{\bar{x}}(y)$ has a maximum in $\bar{x}$ (and, similarly, with "maximum" replaced by "minimum" twice; see Figure 13).

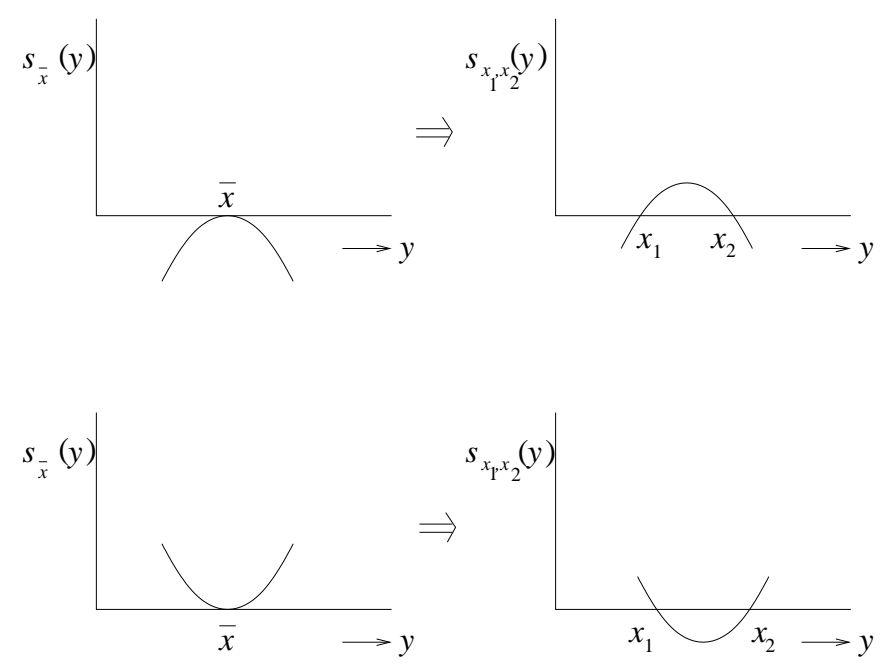

Fig. 13. Deformation of the graph of $s_{\bar{x}}(y)$ into that of $s_{x_{1}, x_{2}}(y)$ : the two generic possibilities

In the case of a maximum, a mutant needs to lie in between $x_{1}$ and $x_{2}$ in order to be successful. Experience and plausibility arguments [68] both tell us that in such a case either $x_{1}$ or $x_{2}$ (or even both) is outcompeted by the successful mutant. So, in case the population stays dimorphic, the distance between the two traits narrows. Repeating the story, we find that both traits move towards $\bar{x}$. One speaks about a converging dimorphism.

In the case of a minimum, a similar analysis demonstrates the diverging character of the dimorphism (at least as long as both $x_{1}$ and $x_{2}$ are still near enough to $\bar{x}$ for a local analysis to be meaningful). 
Now note that the case of a maximum corresponds to $\bar{x}$ being an ESS, i.e., $c_{22}<0$ and that then the mutual invasibility condition $c_{11}>-c_{22}$ entails that $c_{11}>0$ so that automatically the Convergence Stability condition $c_{11}>c_{22}$ holds. We summarize our conclusion for this case: if $\bar{x}$ is CSS, i.e., both ESS and Convergence Stable, then mutual invasibility leads to a converging dimorphism, so dimorphism is a temporary phase in the adaptive approach of $\bar{x}$.

If $\bar{x}$ is an evolutionary repellor, it makes no sense to investigate what happens when $\bar{x}$ is approached monomorphically. In principle it could be that $\bar{x}$, however, is approached by way of a converging dimorphism. But above we found that this is actually impossible.

The remaining case of mutual invasibility, when $\bar{x}$ is Convergence Stable but not ESS, is very interesting: a trait substitution sequence can bring us to a neighbourhood of $\bar{x}$ and then the neighbourhood is left again by way of a diverging dimorphism. A singular point $\bar{x}$ such that $c_{11}>c_{22}>0$ is called a branching point to indicate the not-justtemporary change from monomorphism to dimorphism. After branching has occurred one needs to base a study of the adaptive dynamics on $s_{x_{1}, x_{2}}(y)$ etcetera (and, strictly speaking, on nonlinear competition models with more than two "species"). See [68].

The distinction between converging and diverging dimorphisms brings a moralic message to those who study the possibility of stable coexistence in models for the competition between populations differing only in a particular trait: one should not lean back once stable coexistence is found, but next check the character: is it converging or diverging? Pugliese [75] did this for coexisting strains of an infectious organism and found that the dimorphism was converging!

We have found four dividing lines for regions in the $\left(c_{11}, c_{22}\right)$-plane corresponding to various types of singular points: $c_{22}=0, c_{11}=c_{22}, c_{11}=0, c_{11}=-c_{22}$. In Figure 14 we present the eight regions and draw in each of them a representative local mutual invasibility plot (that is, a PIP with its mirror image under reflection in the diagonal superimposed). The arrows indicate in which direction the trait(s) will move (along the diagonal in case of monomorphism and away or towards the diagonal in case of dimorphism). To stress once more that an ESS is a steady state, but not necessarily an attractor, we point at the region $c_{11}<c_{22}<0$, where $\bar{x}$ is indeed an ESS, but also a repellor for the adaptive dynamics. Nowak \& Sigmund $[73,53]$ have aptly called such a configuration a "Garden of Eden": the only way to get there is by creation and the slightest perturbation that brings you out of this state triggers a gradual but inescapable expulsion.

EXERCISE 7.1. Reconsider the case $s_{x}(y)=r(y, \bar{I}(x))$ with one-dimensional $I$ treated in Section 5. First show that $\bar{x}$ is a singular point if and only if $\frac{d \bar{I}}{d x}(\bar{x})=0$. Next show that necessarily $c_{22}=-c_{11}$ so that either $\bar{x}$ is CSS as well as ESS (and a border line case for mutual invasibility) or a non-steady and repelling state (which only has significance as a "separatrix" for domains of attraction).

EXERCISE 7.2. The evolution of virulence is an intriguing topic. Moreover, it is of great relevance for the quality of human life on earth [23]. A sound strategy is to start analysing a deliberate oversimplification, delaying the consideration of subtle issues till after one has fully understood the caricature. This exercise considers only the caricature, while 


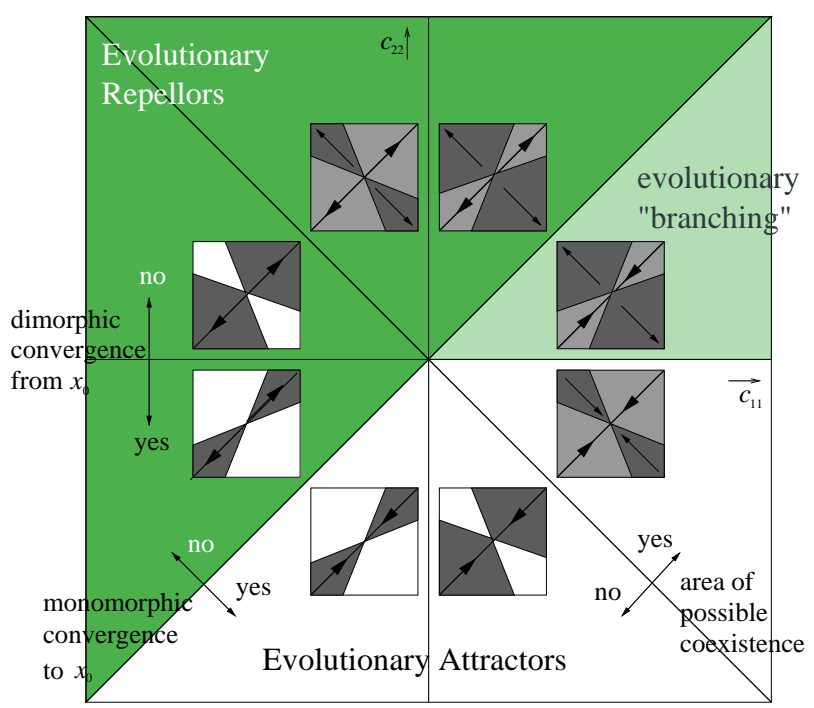

Fig. 14. Classification of singular points. In the eight mutual invasibility plots, regions in which either $s_{x}(y)$ or $s_{y}(x)$ is positive are shaded light grey (and readers are invited to sort out for each such region which of the two is actually positive, accepting the authors apologies for the lack of colours which would have made such an exercise superfluous), regions in which both are positive are shaded dark grey and regions in which both are negative are white. Arrows indicate the direction of adaptive dynamical trait change.

inviting the reader to consult $[23,75]$ for further inspiration, including a wealth of references.

Concerning demography we assume that there is a constant population birth rate $B$ and that individuals live for an exponentially distributed, with parameter $\mu$, period of time. Let $S$ denote the size of the subpopulation of susceptibles and $I$ that of the subpopulation of infectious individuals. Assume that all newborns are susceptible. Assume transmission occurs at per couple rate $\beta$ (so, according to the Law of Mass Action). Assume that an infected individual is immediately infectious and that the infectious period is exponentially distributed with parameter $\gamma$. In low spirits we assume that the infection inevitably leads to death (this will facilitate the interpretation of the trade-off curve of Figure 15; an alternative is to assume that at the end of the infectious period permanent immunity sets in, while speculating about how the immune system will react to increased reproduction of the agent within the host).

Together these assumptions lead to the system of two differential equations:

$$
\begin{aligned}
& \frac{d S}{d t}=B-\mu S-\beta S I, \\
& \frac{d I}{d t}=\quad-\mu I+\beta S I-\gamma I .
\end{aligned}
$$

In the absence of the infection (i.e., for $I=0) S$ settles at $\frac{B}{\mu}$. What happens if we introduce an infected individual into such a "virgin" situation? 
If we introduce an infected individual in a population with susceptible subpopulation size $S$ it transmits, according to the model, the infection to, on average

$$
R_{0}(S)=\frac{\beta S}{\gamma+\mu}
$$

individuals (indeed, the expected duration of its infectious period is $\frac{1}{\gamma+\mu}$ and during this period it transmits the infection at a rate $\beta S)$. When the basic reproduction ratio

$$
R_{0}\left(\frac{B}{\mu}\right)=\frac{\beta B}{\mu(\gamma+\mu)}
$$

exceeds one, the infection becomes endemic. In the endemic state the susceptible subpopulation settles necessarily at $\bar{S}$ such that

$$
R_{0}(\bar{S})=1
$$

so that explicitly we have

$$
\bar{S}=\frac{\gamma+\mu}{\beta} .
$$

Now assume that the strain of the infective agent is characterized by the parameters $\beta$ and $\gamma$, with $\beta$ related to $\gamma$ according to the trade-off curve depicted in Figure 15.

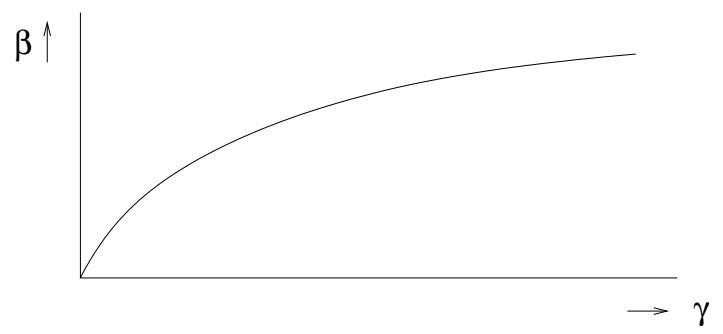

Fig. 15. An increase in infectivity $\beta$ can only be achieved at the expense of a shortening of the infectious period

Finally, if you prefer to consider the case where individuals become immune at the end of the infectious period, assume complete cross-immunity (i.e., having had an infection with some strain yields complete immunity to infection with any other strain).

(i) Explain the rationale underlying Figure 15. (Hint: what effects can increased reproduction within the host have?)

(ii) Show that

$$
s_{x}(y)=\beta(y) \bar{S}_{x}-\mu-y .
$$

(iii) Draw the graph of $\gamma \mapsto \bar{S}_{\gamma}$.

(iv) Construct the corresponding PIP.

(v) Do you find adaptive dynamics towards intermediate virulence? (How would you interpret "virulence"? Do you find the result intuitively obvious in view of Figure 15?)

(vi) Do you find minimization of $\bar{S}_{\gamma}$ and maximization of $R_{0, \gamma}\left(\frac{B}{\mu}\right)$ ? Did you guess this beforehand? 
EXERCISE 7.3 (Predator mediated coexistence). Here is an exercise that I set out to make myself, but that I failed to bring to a satisfactory end. I am inclined to blame lack of time, but this may simply reflect unwarranted optimization of ego. Anyhow, it is a reasonable problem that may, or may not, have a reasonable solution, so deserves to be presented as a challenge.

We return to the consumer-substrate interaction in the chemostat as described in Section 4, but introduce a predator that feeds on the consumer. The system (4.2) is accordingly extended to a three-dimensional system. If, for simplicity, we take a linear up-take rate $g$ and, likewise, a linear functional response to prey concentration, this system reads

$$
\begin{array}{llll}
\frac{d S}{d t}= & D S_{0} & -D S & -S X, \\
\frac{d X}{d t}= & -D X & +\eta S X & -\theta X Y, \\
\frac{d Y}{d t}= & -D Y & +\xi \theta X Y,
\end{array}
$$

(here $X$ has been scaled to make the coefficient of $S X$ in the equation for $\frac{d S}{d t}$ equal to 1 ).

(i) Verify that $S(t)+\frac{1}{\eta} X(t)+\frac{1}{\eta \xi} Y(t) \rightarrow S_{0}$ for $t \rightarrow \infty$.

(ii) Repeat the analysis of Section 4 for the situation without a predator, but now in the $R_{0}$-spirit of the preceding Exercise 7.2. In particular, motivate the definition of

$$
R_{0}(S)=\frac{\eta S}{D}
$$

and verify that the consumer will persist if and only if

$$
R_{0}\left(S_{0}\right)>1
$$

while, if it persists, it steers the substrate concentration to $\bar{S}$ defined by

$$
R_{0}(\bar{S})=1
$$

Finally, compute $\bar{S}$ and $\bar{X}$ explicitly.

(iii) From the point of view of the predator, the environmental condition is fully determined by the consumer concentration $X$. Explain the meaning of the definition

$$
\tilde{R}_{0}(X)=\frac{\xi \theta X}{D}
$$

the condition

$$
\tilde{R}_{0}(\bar{X})>1
$$

and the characterization

$$
\tilde{R}_{0}(\tilde{X})=1
$$

Compute $\tilde{X}$ explicitly.

(iv) With the level at which the consumer stabilizes being determined by predation, the steady substrate level must be determined by putting the expression for $\frac{d S}{d t}$ equal to zero. Verify that this leads to

$$
\tilde{S}=\frac{S_{0}}{1+(\xi \theta)^{-1}} .
$$


(v) When the predator is present, (7.16) needs amendment, as then the environmental condition of the consumer is determined by the two quantities $S$ and $Y$. Motivate the definition

$$
R_{0}(S, Y)=\frac{\eta S}{D+\theta Y}
$$

and solve the equation

$$
R_{0}(\tilde{S}, Y)=1
$$

Call the solution $\tilde{Y}$. Verify that $(\tilde{S}, \tilde{X}, \tilde{Y})$ is a steady state of the system $(7.15)$.

This ends the ecological analysis. (Of course we should check that $(\tilde{S}, \tilde{X}, \tilde{Y})$ is an attractor. All too often in an adaptive dynamics study this step is omitted. Since a beginner's guide should also expose the sins, we follow that reprehensible tradition. Let it serve as an invitation to the next generation to do better.) Now assume that some trait of the consumer may change by mutation and that this is expressed in a change of the parameters $\eta$ and $\theta$. In particular, biochemical energy or machinery may be either spended on improving feeding or on improving defence against predators. So if $\eta$ increases, so does $\theta$ : there is a trade-off. To be specific, consider $\theta$ as specifying the trait and assume $\eta=f(\theta)$ with $f$ describing the trade-off relation. We rewrite (7.23) as

$$
R_{0}(\theta ; S, Y)=\frac{f(\theta) S}{D+\theta Y} .
$$

In earlier examples and in the theory of Section 7 we have based our evolutionary considerations on the real time population growth rate

$$
r(\theta ; S, Y)=-D+f(\theta) S-\theta Y
$$

but the generation growth factor (7.25) carries exactly the same information:

$$
\operatorname{sign} r(\theta ; S, Y)=\operatorname{sign} \ln R_{0}(\theta ; S, Y)=\operatorname{sign}\left(R_{0}(\theta ; S, Y)-1\right)
$$

In this particular case (7.26) is easier to work with. (But in the next section we will see that sometimes $R_{0}$ is to be preferred.)

(vi) Show that singular points are characterized by the equation

$$
f^{\prime}(\theta) \tilde{S}_{\theta}-\tilde{Y}_{\theta}=0
$$

and that, given a solution $\bar{\theta}$ of $(7.28)$,

$$
\begin{gathered}
c_{22}=f^{\prime \prime}(\bar{\theta}) \tilde{S}_{\bar{\theta}}, \\
c_{11}=f(\bar{\theta})\left(\frac{d^{2}}{d \theta^{2}} \tilde{S}_{\theta}\right)_{\theta=\bar{\theta}}-\bar{\theta}\left(\frac{d^{2}}{d \theta^{2}} \tilde{Y}_{\theta}\right)_{\theta=\bar{\theta}} .
\end{gathered}
$$

(vii) Now here, at last, comes the only difficult part of this exercise: establish conditions on $f$ such that a branching point exists (i.e., a solution $\bar{\theta}$ of (7.28) such that $c_{11}>c_{22}>0$, with $c_{11}$ and $c_{22}$ given by (7.30) and (7.29). The motivation for this exercise derives from the ecological literature, where predator mediated coexistence is found: for certain combinations $\left(\eta_{1}, \theta_{1}\right)$ and $\left(\eta_{2}, \theta_{2}\right)$ consumers of type 1 and type 2 can coexist by virtue of mutual invasibility. The idea is that the better competitor for substrate suffers more from the predator and that a perfect balance can be achieved. But is this a converging or a diverging dimorphism? In the first case we should dismiss the 
phenomenon as being unimportant, in the second case we should take it serious. To you, dear reader, the task to find out.

8. The timing of reproduction. A semelparous organism reproduces only once in its life. Examples include many plants, pacific salmon, cicadas and other, so-called periodic insects $[3,4]$. If the life of any organism deserves to be called a cycle, it are these.

When every year brings only one reproduction opportunity, say in the spring, a "decision" has to be made concerning either reproducing "now", with the inevitable consequence of death, or to delay reproduction another year, with the doubtful necessity of having to survive the winter. In the caricatural spirit of these notes we shall investigate how natural selection decides. The analysis allows us to demonstrate various methodological points and to illustrate several general insights, related to the following questions:

- how to analyse (steady states of) structured population models?

- what difference can the dimension of the environmental interaction variable $I$ make?

- how does adaptive dynamics relate to the optimization of a fitness measure?

- how important is the precise nature of density dependence?

- what is the ideal free distribution?

This section is inspired by, but differs substantially from, the paper [51]. We work on a discrete time basis and assume a census is taken every spring, just before reproduction takes place. We severely limit the spectrum of possible choices: a one year old individual can reproduce or delay reproduction, but a two year old individual has to reproduce. In other words, to be annual or to be biennial, that's the question.

The two-dimensional system of recurrence relations

$$
\begin{aligned}
& N_{1}(t+1)=s_{0}\left(\gamma f_{1} N_{1}(t)+f_{2} N_{2}(t)\right), \\
& N_{2}(t+1)=s_{1}(1-\gamma) N_{1}(t),
\end{aligned}
$$

describes this situation. Here $N_{i}$ is the density of $i$-year old individuals, $s_{i}$ are survival probabilities and $f_{i}$ are fecundities. The parameter $\gamma$ is the trait that is supposed to be subject to natural selection and mutation. It is the probability that a one year old individual will reproduce, so $0 \leq \gamma \leq 1$ and $\gamma=1$ describes an annual species while $\gamma=0$ corresponds to a biennial species.

A schematic representation of the bookkeeping aspects of the model is provided in Figure 16. Our analysis proceeds in steps. First we consider the linear model in which all parameters are fixed. Next we consider nonlinear models in which the survival probabilities $s_{i}$ are density dependent. We look at three versions, two with a one-dimensional $I$ and one with a two-dimensional $I$. We detail how steady states can be determined (and how the fact, that there is only one possible state-at-birth, makes this relatively simple). Finally we investigate how $\gamma$ will evolve. In the cases with one-dimensional $I$ we find, as we should, pessimization. But we show how a more optimistic reformulation takes the form of the optimization of a fitness measure. We emphasize that it is the nonlinearity, the way density dependence acts, that determines which fitness measure is appropriate (thus echoing the main message of $[70,29]$ ). Moreover, when $I$ is higher-than-one dimensional the whole idea of a fitness measure is simply nonsense. We demonstrate how 


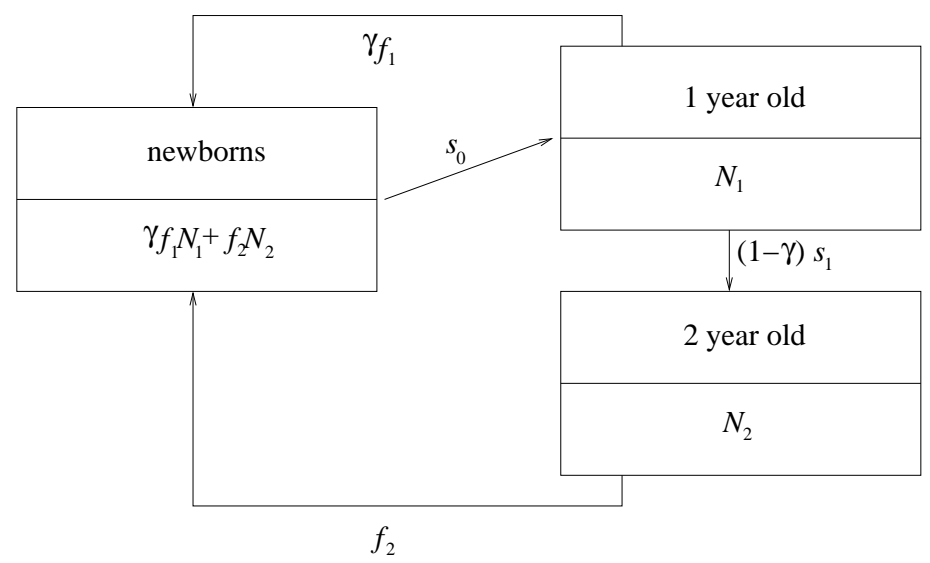

Fig. 16. The life cycle of a semelparous organism that can live for at most two years

natural selection may have an "equalizing" effect, in particular how at singular points different pathways to reproduction should necessarily be equally attractive (this goes by names like "the ideal free distribution" and "the marginal value theorem" but I like to call it "the principle of indifference").

8.1. Linear population dynamics. A newborn survives the first winter with probability $s_{0}$ and, when it delays reproduction, the second with probability $s_{1}$. So it produces on average

$$
R_{0}=s_{0}\left(\gamma f_{1}+(1-\gamma) s_{1} f_{2}\right)
$$

offspring. So when we look at growth in generation perspective, we have an explicit expression for the growth factor, the basic reproduction ratio $R_{0}$.

The growth (or decline) in real time is described by the Leslie matrix

$$
L=\left(\begin{array}{cc}
\gamma f_{1} s_{0} & f_{2} s_{0} \\
(1-\gamma) s_{1} & 0
\end{array}\right) .
$$

One finds the eigenvalues as roots of the characteristic equation

$$
\lambda^{2}-\gamma f_{1} s_{0} \lambda-(1-\gamma) f_{2} s_{1} s_{0}=0 .
$$

but, remarkably, the more informative way (in particular for the higher dimensional analogue, which cannot be solved explicitly) of writing this equation is

$$
1=\frac{\gamma f_{1} s_{0}}{\lambda}+\frac{(1-\gamma) f_{2} s_{1} s_{0}}{\lambda^{2}}
$$

The point is that the right hand side equals $R_{0}$ for $\lambda=1$ and defines a monotone decreasing function of real $\lambda$, which vanishes for $\lambda \rightarrow \infty$ while going to $\infty$ for $\lambda \downarrow 0$. So it follows at once (you are invited to draw some pictures yourself) that (8.5) has a unique positive root, which we call $\lambda_{d}$, and that

$$
\operatorname{sign}\left(\lambda_{d}-1\right)=\operatorname{sign}\left(R_{0}-1\right) .
$$


By either invoking the Perron-Frobenius theorem or by estimating the right hand side of (8.5) for complex $\lambda$, one concludes that for $\gamma>0$

$$
|\lambda|<\lambda_{d} \text { if } \lambda \text { is eigenvalue of } L \text { and } \lambda \neq \lambda_{d}
$$

so that $\lambda_{d}$ is indeed the dominant eigenvalue of $L$ (whence the index $d$ ). But note that for $\gamma=0$ the second eigenvalue equals $-\lambda_{d}$ and that then there are two reproductively isolated year classes, those reproducing in odd years and those reproducing in even years. In the jargon of positive matrices we say that $L$ is irreducible but not primitive. In biological terms we say that the population decomposes into two independent subpopulations.

The population will grow (assuming $\lambda_{d}>1$ ) like

$$
\lambda_{d}^{t}=\mathrm{e}^{t \ln \lambda_{d}}
$$

in the course of time $t$, so with rate $\ln \lambda_{d}$. The equality (8.6) shows that $R_{0}$ and $\lambda_{d}$ are equivalent as indicators of population growth or decline.

When (8.7) is satisfied the population composition will, in the course of time, stabilize and take the "shape" of the eigenvector corresponding to $\lambda_{d}$ (that is, the ratio of the sizes of the various age groups resembles more and more the ratio of the corresponding components of the dominant eigenvector). The second component of the identity $L N=$ $\lambda_{d} N$ reads $s_{1}(1-\gamma) N_{1}=\lambda_{d} N_{2}$ so the vector

$$
\left(\begin{array}{c}
\lambda_{d} \\
s_{1}(1-\gamma)
\end{array}\right)
$$

is such an eigenvector.

In the present case we can of course find an explicit expression for $\lambda_{d}$ by solving (8.4). However, in general this is impossible and one has to rely on the qualitative arguments concerning (8.5) (or, equivalently, the theory of positive matrices and positive operators) to establish the existence and uniqueness of $\lambda_{d}$ such that (8.7) holds. In contrast, the formula (8.2) for $R_{0}$ can be extended easily to Leslie matrices of arbitrary dimension.

8.2. Nonlinear population dynamics. There are various ways in which feedback may act. Here we restrict to density dependent survival, assuming the fecundities $f_{0}$ and $f_{1}$ are not affected by density. But even then there is a host of possibilities. We classify according to the number of components of $I$ and present two examples with $\operatorname{dim} I=1$ and one with $\operatorname{dim} I=2$. Moreover, we restrict our attention to steady states and, objectionable as it may be, do not check whether these steady states are indeed attractors for the population dynamics (readers are invited/encouraged to be more scrupulous).

If $I$ is a scalar, the first step is to determine the steady state value $\bar{I}$ by solving the equation

$$
R_{0}(I)=1 \text {. }
$$

In the second step one employs $(8.9)$ for $\lambda_{d}=1$ (since $R_{0}=1$ ) to conclude that the steady population state has the form

$$
c\left(\begin{array}{c}
1 \\
s_{1}(1-\gamma)
\end{array}\right)
$$


with $c$ still to be determined. The scalar $c$ is then determined by consistency: if (8.11) is substituted in the expression for $I$, the outcome should be $\bar{I}$.

We now illustrate this systematic general procedure by way of two concrete examples.

8.2.A.1. Nursery competition. Assume $s_{0}=s_{0}(I)$ with

$$
I=\gamma f_{1} N_{1}+f_{2} N_{2}
$$

(cf. Figure 16) and $s_{0}$ a decreasing function with $s_{0}(0)=p$ and $s_{0}(I) \downarrow 0$ for $I \rightarrow \infty$. Provided $R_{0}(0)=p\left(\gamma f_{1}+(1-\gamma) s_{1} f_{2}\right)>1$ the equation (8.10) has a unique solution $\bar{I}$. The unknown $c$ in (8.11) is determined from

$$
\bar{I}=c\left(\gamma f_{1}+(1-\gamma) s_{1} f_{2}\right)
$$

and hence can be computed explicitly (in terms of model parameters and the only implicitly known $\bar{I}$ ). Presumably the steady state is stable in the Beverton-Holt case $s_{0}(I)=p(1+q I)^{-1}$ but it is bound to be unstable for a non-negligible parameter range in the Ricker case $s_{0}(I)=p \exp (-q I)$. As mentioned above, we do not attempt to determine (or even just delineate) this parameter range.

8.2.A.2. Uniform winter competition. Assume both $s_{0}$ and $s_{1}$ depend on $I$ and, in fact, in exactly the same manner, i.e., $s_{0}(I)=s_{1}(I)=s(I)$ with $s$ a decreasing function. Assume that $I$ is the density of those that overwinter, i.e.,

$$
I=\gamma f_{1} N_{1}+f_{2} N_{2}+(1-\gamma) N_{1} .
$$

Again monotonicity arguments show that (8.10) has a unique positive solution $\bar{I}$ provided $R_{0}(0)>1$ and $R_{0}(\infty)<1$, while substitution of (8.11) into (8.14) for $I=\bar{I}$ yields the "explicit" expression

$$
c=\frac{\bar{I}}{\gamma f_{1}+f_{2}(1-\gamma) s(\bar{I})+1-\gamma} .
$$

As a minor side-remark we mention that the structure of the model remains exactly the same if we allow $s_{0}$ and $s_{1}$ to differ by a fixed (i.e., $I$-independent) factor and if we introduce fixed weight factors for the terms $\gamma f_{1} N_{1}+f_{2} N_{2}$, corresponding to newborns, and $(1-\gamma) N_{1}$, corresponding to one-year-old individuals, in the expression (8.14) for $I$.

8.2.B. Age-specific winter competition. Assume that both $s_{0}$ and $s_{1}$ are under the influence of population density, but that $s_{0}$ is affected only by the density of young of the year, while $s_{1}$ is determined by competition of overwintering one-year-old individuals among themselves. We then put

$$
I_{1}=\gamma f_{1} N_{1}+f_{2} N_{2}, \quad I_{2}=(1-\gamma) N_{1},
$$

and let $s_{0}$ depend, in a monotone decreasing manner, on $I_{1}$ and $s_{1}$, in a similar monotone way, on $I_{2}$. The key difference is that now the equation (8.10) is one equation in two unknowns. As a consequence the determination of $\bar{I}$ and $c$ has to proceed by analysing a fully coupled problem (while before we had a one-way coupling only: the equation for $c$ involved $\bar{I}$, but the one for $\bar{I}$ was independent of $c$ ).

This problem is (8.19) below. It is obtained by combining (8.2)-(8.10) and (8.11)(8.16). But first we digress on the general structure. 
In [28] ] it is shown how the steady state problem for a general class of physiologically structured population models can be brought into the form

$$
L(I) b=b, \quad I=G(I) b,
$$

where $b$ is the vector (assuming there are at most finitely many states-at-birth possible) of steady birth rates and $I$ is the vector (assuming there are finitely many components only) of steady environmental interaction variables. Discretization (of states-at-birth and of environmental conditions) may be needed as a preparatory step to achieve the finiteness. The matrices $L(I)$ and $G(I)$ can be constructed numerically by solving ordinary differential equations describing the maturation, survival and reproduction of individuals, given the environmental conditions, see [58]. Note that the problem is linear in $b$. The basic reproduction ratio $R_{0}(I)$ is by definition the dominant eigenvalue of the positive matrix $L(I)$. Since the interpretation requires $b$ to be positive, one can rewrite $L(I) b=b$ in the form $R_{0}(I)=1$ and $b=c b_{0}(I)$ where $b_{0}(I)$ is a, conveniently normalized, eigenvector of $L(I)$ corresponding to $R_{0}(I)$. Thus (8.17) becomes

$$
R_{0}(I)=1, \quad I=c H(I),
$$

where $H(I)=G(I) b_{0}(I)$. These are $\operatorname{dim} I+1$ equations in as many unknowns. Whenever all individuals are born with the same state-at-birth, the problem (8.17) has the structure (8.18) right from the start, as then $b$ as well as $L(I)$ are scalars (while $G(I)$ has the dimension of $I$ ).

In the case of the present example (8.18) takes the form

$$
\begin{aligned}
& s_{0}\left(I_{1}\right)\left\{\gamma f_{1}+(1-\gamma) s_{1}\left(I_{2}\right) f_{2}\right\}=1, \\
& I_{1}=c\left(\gamma f_{1}+(1-\gamma) s_{1}\left(I_{2}\right) f_{2}\right), \\
& I_{2}=c(1-\gamma),
\end{aligned}
$$

which are three equations in the three unknowns $I_{1}, I_{2}$ and $c$. It is easy enough to reduce the system to one equation in one unknown, but, as our main interest is in adaptive dynamics, the characterization of steady states by (8.19) suffices.

8.3. Adaptive dynamics of $\gamma$. A special feature of the model is that the growth indicator $R_{0}$ is linear in the trait $\gamma$, cf. (8.2). As a consequence the selection gradient, when computed in terms of $R_{0}$ rather than $\lambda_{d}$, does not depend on $\gamma$ explicitly. This selection gradient equals

$$
\left.\frac{\partial R_{0}}{\partial \gamma}(I, \gamma)\right|_{I=\bar{I}(\gamma)}=s_{0}\left(f_{1}-s_{1} f_{2}\right)
$$

where at the right hand side we did not yet express in the notation that $s_{0}$ and $s_{1}$ depend on $\bar{I}(\gamma)$, since the way they do differs a bit from example to example. The two terms in (8.20) correspond to the two "pathways" to reproduction and the fact that one goes at the expense of the other is reflected in the opposite signs. We now consider the three examples in turn.

8.3.A.1. Nursery competition. When only $s_{0}$ depends on $I$ the basic reproduction ratio factorizes in the product of a function of $I$ and a function of $\gamma$

$$
R_{0}(\gamma, I)=s_{0}(I) \rho(\gamma)
$$


with

$$
\rho(\gamma):=\gamma f_{1}+(1-\gamma) s_{1} f_{2}
$$

The steady state equation $R_{0}=1$, cf. (8.10), then implies that

$$
s_{0}\left(\bar{I}_{\gamma}\right)=\frac{1}{\rho(\gamma)} .
$$

Consequently we have that

$$
R_{0}\left(\gamma_{\mathrm{inv}}, \bar{I}_{\gamma_{\mathrm{res}}}\right)=\frac{s_{0}\left(\bar{I}_{\gamma_{\mathrm{res}}}\right)}{s_{0}\left(\bar{I}_{\gamma_{\mathrm{inv}}}\right)}=\frac{\rho\left(\gamma_{\mathrm{inv}}\right)}{\rho\left(\gamma_{\mathrm{res}}\right)}
$$

and the pessimization principle, that $s_{0}(I)$ is minimized in the course of the adaptive dynamics, allows the equivalent reformulation that $\rho(\gamma)$ is maximized.

Much of the standard literature on evolutionary ecology, such as [77, 10,81], introduces an ad-hoc version of this result: one declares $R_{0}$ to be a fitness measure which is maximized by evolution. Even experts have been puzzled by the paradoxical nature of this formulation: how can a quantity be maximized that, in steady state, has to be equal to one? The unambiguous and careful adaptive dynamics analysis, based on the fundamental notion of invasibility, clarifies the situation at once: whenever $R_{0}$ can be factorized as in (8.21), the factor $\rho$, which tells us how $R_{0}$ depends on the trait, will be maximized (with the inevitable consequence that the second factor, which tells us how $R_{0}$ depends on the environmental conditions, will be minimized, since the product is fixed at the value 1). One lesson to be learned is that notation is extremely important: if one always just writes $R_{0}$, without indicating either the trait or the steady environmental conditions that should be specified, it is impossible to formulate (8.24) and confusion is the inescapable result.

We present the conclusion for model A.1 in Figure 17.

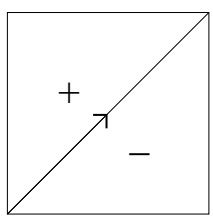

$f_{1}>s_{1} f_{2}$

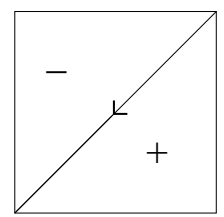

$f_{1}<s_{2} f_{2}$

Fig. 17. The two possible PIP's for the nursery competition model A.1. In the evolutionary end the species will either be a strict annual (left) or a strict biennial (right) reproducer.

8.3.A.2. Uniform winter competition. The key point of this example is that now the quantity $\lambda_{d}$ factorizes into the product of an $I$-dependent factor and a $\gamma$-dependent factor. To see this, we write the characteristic equation (8.5) as

$$
1=\frac{s(I)}{\lambda} \gamma f_{1}+\left(\frac{s(I)}{\lambda}\right)^{2}(1-\gamma) f_{2}
$$

and define $\sigma=\sigma(\gamma)$ to be the unique positive root of the equation 


$$
1=\frac{\gamma f_{1}}{\sigma}+\frac{(1-\gamma) f_{2}}{\sigma^{2}}
$$

It follows that

$$
\lambda_{d}(\gamma, I)=s(I) \sigma(\gamma)
$$

and that adaptive dynamics corresponds to the maximization of $\sigma(\gamma)$ and, simultaneously/equivalently, to the minimization of $s(I)$. (Indeed, just note that

$$
\lambda_{d}\left(\gamma_{\mathrm{inv}}, \bar{I}_{\gamma_{\mathrm{res}}}\right)=\frac{s\left(\bar{I}_{\gamma_{\mathrm{res}}}\right)}{s\left(\bar{I}_{\gamma_{\mathrm{inv}}}\right)}=\frac{\sigma\left(\gamma_{\mathrm{inv}}\right)}{\sigma\left(\gamma_{\mathrm{res}}\right)}
$$

and use that necessarily $\sigma\left(\gamma_{\text {inv }}\right) / \sigma\left(\gamma_{\text {res }}\right)<1$, for $\gamma_{\text {inv }} \neq \gamma_{\text {res }}$, if $\gamma_{\text {res }}$ is such that $\sigma$ has a strict maximum for that value of $\gamma$ ). For this result too there is a much used ad hoc version in the standard literature on evolutionary ecology. We emphasize that the key point is the factorization (8.27), but that it is irrelevant whether or not we have an explicit expression for $\sigma(\gamma)$ (here we can obtain such an expression by solving (8.26), but in higher dimensional analogues we will not be able to do so). By noting that $\sigma(1)=f_{1}$, $\sigma(0)=\sqrt{f_{2}}$, and by excluding the possibility that $\sigma^{\prime}(\gamma)=0$ (or, in other words, by showing that $\sigma$ is monotone) one finds the conclusion for model A.2 as presented in Figure 18.
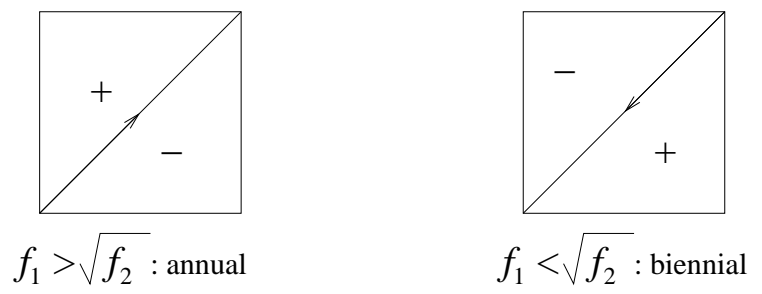

Fig. 18. The two possible PIP's for the uniform winter competition model A.2. Again we find that in the evolutionary end the species will either be a strict annual or a strict biennial reproducer. But note that the criterion used to decide which will be the case differs from that obtained for model A.1.

The conclusion of part A is that a strict dichotomy exists: either the species becomes an annual reproducer or it becomes a biennial reproducer. However, the outcome does depend on the precise way in which density dependence acts: if either $\sqrt{f_{2}}<f_{1}<s_{1}(0) f_{2}$ or $s_{1}(0) f_{2}<f_{1}<\sqrt{f_{2}}$ the two versions of density dependence (which are by far not the only conceivable ones) give different predictions. As often population biologists do not know very well how density dependence acts, they want results which are independent of density dependence. The examples above show that this is an illusion. To pretend that one can get conclusions by "choosing" a fitness measure is ostrich policy, as in fact this boils down to an implicit choice for the mechanism of density dependence. The two examples were concocted such that either $R_{0}$ or $\lambda_{d}$ could be factorized, but this was mainly done to establish the connection with the ad hoc methods in the standard literature on evolutionary ecology. The key feature which entails a pessimisation/optimisation principle is the one-dimensionality of $I$. So let us now turn to the example where this is violated. 
8.3.B. Age-specific winter competition. Repeating the expression (8.20) for the selection gradient here in the form

$$
\left.\frac{\partial R_{0}}{\partial \gamma}(I, \gamma)\right|_{I=\bar{I}(\gamma)}=s_{0}\left(\bar{I}_{1, \gamma}\right)\left(f_{1}-s_{1}\left(\bar{I}_{2, \gamma}\right) f_{2}\right)
$$

we see that, even though the right hand side doesn't depend on $\gamma$ explicitly, there is a possibility for an internal (i.e, $\gamma \neq 0,1$ ) singular point, since it may happen that for some $\gamma$ we have the identity

$$
s_{1}\left(\bar{I}_{2, \gamma}\right) f_{2}=f_{1},
$$

meaning that the two different reproduction pathways are precisely equally profitable. In such a rather special situation all $\gamma$ actually perform equally well (see (8.2) and note that the right hand side is independent of $\gamma$; so if you try to measure in this situation the selection pressure on $\gamma$ you don't find any!). (Attentive readers may wonder why we skipped the possibility $s(\bar{I}) f_{2}=f_{1}$ in model A.2? Perhaps they then now realize that it yields exactly the borderline case separating the two generic cases depicted in Figure 18 from one another.)

In evolutionary ecology it happens rather often that a population of individuals tunes the environmental conditions such that the various options that are open to the individuals become equally attractive. The name "ideal free distribution" is used to denote this phenomenon [57]. The "principle of indifference" is an alternative denomination that seems to capture the key point rather well. In economy, and consequently in optimal foraging theory and other areas of behavioural ecology, one uses the name "marginal value theorem" to denote that an optimum is to be found there where infinitesimal re-allocations don't make any difference.

Well, but does a singular point characterized by (8.30) indeed exist? If so, is it unique?

The inequality

$$
s_{1}(0) f_{2}>f_{1}>1
$$

clearly is a necessary condition on the model ingredients in order that a solution of (8.30) exists, so we assume it is satisfied. The three step procedure

- solve $\bar{I}_{2}$ from $f_{2} s_{1}\left(\bar{I}_{2}\right)=f_{1}$

- solve $\bar{I}_{1}$ from $f_{1} s_{0}\left(\bar{I}_{1}\right)=1$

- define

$$
\bar{\gamma}=1-\frac{f_{1} \bar{I}_{2}}{\bar{I}_{1}}
$$

and check that $0<\bar{\gamma}<1$

yields the answer to the two questions, as we now explain. The first step guarantees that the condition (8.30) for a singular point is satisfied. The second step guarantees that $R_{0}=1$ (given that (8.30) holds). In the third step the feedback condition $\bar{I}=c H(\bar{I})$ (see (8.18)) is considered but now, since $\bar{I}$ is already determined, as two equations in the two unknowns $c$ and $\gamma$ (note that $H$ depends on $\gamma$, even though this isn't expressed in the notation). Written out in full the feedback condition reads

$$
\bar{I}_{1}=c\left(f_{1} \gamma+f_{2}(1-\gamma) s_{1}\left(\bar{I}_{2}\right)\right)=c f_{1}, \quad \bar{I}_{2}=c(1-\gamma)
$$


where we have used (8.30) to simplify the right hand side of the first equation. Eliminating $c$ and solving for $\gamma$ we obtain (8.32).

As the procedure is in terms of conditions which are not only sufficient but also necessary and as it yields at most one $\gamma$, the uniqueness question is settled. The condition $0<\bar{\gamma}<1$ amounts to the inequality

$$
f_{1} \bar{I}_{2}<\bar{I}_{1}
$$

which can be checked once steps 1 and 2 are executed.

The condition $s_{1}(0) f_{2}>f_{1}$, which is part of (8.31), is the condition that the selection gradient (8.29) is negative in $\gamma=1$ or, in other words, that the "annual" strategy is a repellor for the adaptive dynamics. Likewise (8.34) is the condition that the selection gradient (8.29) is positive in $\gamma=0$ or, in other words, that the "biennial" strategy is a repellor as well. The simplest arguments to substantiate this last claim is an indirect one based on the intermediate value theorem: as the selection gradient can change sign in the interval $(0,1)$ at most once, its sign at the end points $\gamma=0$ and $\gamma=1$ is opposite if and only if it changes sign.

So if $\bar{\gamma}$ exists (i.e., if (8.34) holds) it must be Convergence Stable! As already noted in the beginning of Subsection 8.3, the selection gradient does not depend explicitly on the trait $\gamma$, since $R_{0}$ is linear in $\gamma$. Accordingly

$$
c_{22}=\frac{\partial^{2} R_{0}}{\partial \gamma_{\text {inv }}^{2}}=0
$$

and we have a borderline case between a converging (to ESS) and a diverging (away from a branching point) dimorphism.

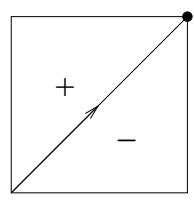

$s_{1}(0) f_{2}<f_{1}$

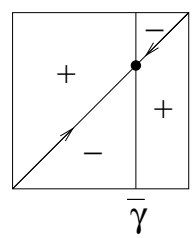

$s_{1}(0) f_{2}>f_{1}$

$\bar{I}_{1}>f_{1} \bar{I}_{2}$

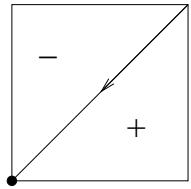

$s_{1}(0) f_{2}>f_{1}$

$\bar{I}_{1}<f_{1} \bar{I}_{2}$

Fig. 19. The three possible PIP's in the case of age-specific winter competition. It is still possible that either the strict annual (left picture) or the strict biennial (right picture) outcompetes all other strategies, but, as the middle picture shows, it is now also possible to have an intermediate CSS.

The linearity in $\gamma$ implies that $c_{22}=0$ is a local reflection of a global phenomenon, the neutrality of all $\gamma$ when the resident has trait $\bar{\gamma}$. In the middle PIP of Figure 19, this manifests itself in the vertical straight line at which the invasion exponent changes sign. But it presents an enigmatic feature: the mutual invasibility near $\bar{\gamma}$ yields a protected dimorphism for which our first order analysis does not establish whether it is converging or diverging!!! Readers are now invited to venture a guess as to what will happen when 
$\gamma$, by a sequence of trait substitutions, comes close to $\bar{\gamma}$. (Please formulate your guess explicitly before reading on.)

Consider a dimorphic population consisting of individuals with trait $\gamma_{1}$ and individuals with trait $\gamma_{2}$. Assume that both $I_{1}$ and $I_{2}$ settle at constant values. Then these should be such that both the $R_{0}$ for the $\gamma_{1}$-subpopulation and the one for the $\gamma_{2}$-subpopulation are equal to one. Since, for given $I_{1}$ and $I_{2}, R_{0}$ is a linear function of $\gamma$, this can only happen if $R_{0}$ is constant as a function of $\gamma$ and this, in turn, can only happen if $I_{2}$ is such that (8.30) holds. In order for the constant value to be equal to one we next should choose $I_{1}$ such that $f_{1} S_{0}\left(I_{1}\right)=1$. In summary, $I_{1}$ and $I_{2}$ should be exactly the $\bar{I}_{1}$ and $\bar{I}_{2}$ obtained in the first two steps of our earlier procedure. It is only in the third step that the dimorphic case differs from the monomorphic case.

In the dimorphic case the feedback condition involves two constants, $c_{1}$ and $c_{2}$, measuring the size of the corresponding subpopulations. (So whereas before we used the feedback condition to determine $c$ and $\gamma$, we now have fixed $\gamma_{1}$ and $\gamma_{2}$ beforehand, but use the two feedback relations to determine the two-dimensional c.) Using for each subpopulation the stable distribution $(8.11)$ we find that the feedback conditions are

$$
\begin{aligned}
& \bar{I}_{1}=f_{1} \gamma_{1} c_{1}+f_{2}\left(1-\gamma_{1}\right) s_{1} c_{1}+f_{1} \gamma_{2} c_{2}+f_{2}\left(1-\gamma_{2}\right) s_{1} c_{2}=f_{1} c_{1}+f_{1} c_{2}, \\
& \bar{I}_{2}=\left(1-\gamma_{1}\right) c_{1}+\left(1-\gamma_{2}\right) c_{2},
\end{aligned}
$$

where we suppressed the dependence of $s_{1}$ on $\bar{I}_{2}$ in the notation and where we used that, according to (8.30), $f_{2} s_{1}=f_{1}$, to simplify the first equation. The solution of this linear system reads

$$
\left(\begin{array}{l}
c_{1} \\
c_{2}
\end{array}\right)=\frac{1}{f_{1}\left(\gamma_{1}-\gamma_{2}\right)}\left(\begin{array}{c}
\left(1-\gamma_{2}\right) \bar{I}_{1}-f_{1} \bar{I}_{2} \\
-\left(1-\gamma_{1}\right) \bar{I}_{1}+f_{1} \bar{I}_{2}
\end{array}\right)
$$

but note that, in order for the explicit expression to be biologically meaningful, the $c_{i}$ thus defined must be positive! Recalling (8.32) we see that this requirement is fulfilled if and only if $\gamma_{1}$ and $\gamma_{2}$ lie at opposite sides of $\bar{\gamma}$. We conclude that a dimorphic population with such a combination of traits can set the environmental conditions in exactly the same way as a monomorphic $\bar{\gamma}$ population (due to the linearity in $\gamma$ the appropriate mixture of $\gamma_{1}$ and $\gamma_{2}$ subpopulations is, when measured in terms of the environmental conditions they generate, the same as a $\bar{\gamma}$ population). Once these environmental conditions are set, there is complete neutrality: all $\gamma$ perform equally well. So, although drift may still occur, there are no further trait substitutions. Assuming that the dimorphic steady state determined above is indeed an attractor for the dimorphic population dynamics, we conclude that, once trait substitutions have brought $\gamma$ close enough to $\bar{\gamma}$ for the other side of $\bar{\gamma}$ to be within mutational reach, it depends on mutational chance effects whether the trait moves on towards $\bar{\gamma}$ : as soon as a mutant "at the other side of $\bar{\gamma}$ " is generated, no further adaptations occur and the population stays dimorphic (here we adhere to the deterministic view based on extremely large population size, i.e., we ignore drift due to demographic stochasticity).

The conclusion of part B is that the spectrum of possibilities widens when the dimension of the environmental condition, as far as it is under the influence of population density by feedback, exceeds one. Even though $R_{0}$ is still monotone as a function of each 
of the components of $I$ separately, there is no pessimization principle. Instead one should use the principle of indifference as a guiding principle for the search for singular points (note of caution: the linearity in $\gamma$ makes our example misleadingly simple; in general one should look for environmental conditions and a trait value as a solution of a coupled system of equations and the indifference refers to infinitesimal changes in the trait only). This reinforces what we said before: the precise form in which density dependence acts is of crucial importance for evolutionary considerations!

8.4. The "resident strikes back" phenomenon. So far we have concentrated on competition under constant (in time) conditions. It is important to realize that, when environmental conditions fluctuate, strategies may be superior that are inferior under constant conditions. This relates to bet hedging. An illustrative example is that seeds of many plants delay germination with non-negligible probability, thus forming a seed bank as a kind of insurance against the bad luck of a year with disastrous weather conditions. Optimal bet hedging under stochastic environmental conditions is a subtle issue [83, 85].

Here we are concerned with fluctuations that are due to density dependence and that have a predictable rhythm. As we work in discrete time, the ensueing periodic environmental condition is still finite dimensional (i.e., completely described by finitely many real numbers). Accordingly it is still easy to compute an invasion exponent.

But a subtle point is that, as observed in Subsection 8.1, a strictly biennial population splits into two reproductively isolated year classes, those reproducing in odd years and those reproducing in even years. Under constant conditions both grow (or decline) at the same rate. But what if the conditions vary periodically? In particular, what if the greatest common divisor of the period of the environmental fluctuations and the length of the life cycle (so two in the biennial case) exceeds one? Then the world as experienced by the various year classes may be quite different. Some may systematically hit the good conditions, while others may be pursued by bad luck. As a consequence, the invasion exponent is year class specific (or, if you prefer, multivalued).

A striking manifestation of this phenomenon occurs if we consider nursery competition between an annual $(\gamma=1)$ and a biennial $(\gamma=0)$. Suppose the annual is the resident and assume $s_{0}$ is such that the size of its cohorts oscillate with period two (for instance, take the Ricker function $s_{0}(I)=\exp (-I)$ then, upon increasing $f_{1}$, the steady state will lose its stability by a period doubling bifurcation and for a substantial window of $f_{1}$ values the population dynamical attractor will be a two-cycle). Suppose $s_{0}$ has a high value in even years and a low value in odd years, then we might call even years good and odd years bad. Consequently the biennial year class that resides in the nursery in even years experiences the world as obliging, while the other year class, in contrast, experiences the world as harsh. A first result is the possibility of "resonance mediated coexistence" of the annual with the even year class of the biennial, under parameter conditions such that the annual would outcompete the biennial in a steady world. But a second result is more striking: if one increases the competitive strength of the biennial, it may happen that the even year class of the biennial, after successful invasion, pesters the annual so much that for it the odd years become the better years. Thus the biennial triggers a phase shift, which brings about its own ruin, as the systematic advantage of the past turns 
into a systematic disadvantage of the future. The success is transitory, rise is followed by downfall, as the resident strikes back by shifting the phase of the feedback generated oscillation in the environmental conditions.

The technical underpinning of the verbal description above is in the paper [72] (also see [71]). It involves the system

$$
\begin{aligned}
& N(t+2)=s_{0}(I(t+1)) f_{1} s_{0}\left(f_{1} N(t)\right) f_{1} N(t), \\
& M(t+2)=s_{0}(I(t+1)) f_{2} s_{1} M(t), \\
& I(t+1)=f_{1} s_{0}\left(f_{1} N(t)\right) f_{1} N(t)+f_{2} s_{1} M(t),
\end{aligned}
$$

which describes transitions in the annual cohort $N$ and the cohort $M$ of one biennial year class, when we step two years ahead in time. The transition from coexistence to the "resident strikes back" situation is by way of an extremely small parameter window of heteroclinic tangle dynamics. If we look at the "resident strides back" situation in $\mathbb{R}^{3}$, i.e., if we include the other biennial year class in our analysis, there is a stable heteroclinic cycle at the boundary of the positive cone. Numerically this cycle appears to be the $\omega$-limit set for all orbits that start in the interior of the cone.

This subsection is a bit of a side-slip. It exposes the importance of resonance and phase for the value of the invasion exponent in case of periodic environmental conditions, especially when the life of the organism considered is itself almost literally a cycle. It shows, by way of example, that a successfully invading mutant may induce an attractor shift of the resident and perish as a result. So it serves as a warning sign: that successful invasion implies either take over or dimorphism is not a hard and fast rule! And, admittedly, the aim of this subsection is also to draw attention to a neat dynamical system, viz. the one generated by $(8.37)$ and its extension to $\mathbb{R}^{3}$.

\section{Concluding remarks (including a glance at the agenda of the advanced).}

Ever since Darwin scientists have aimed to describe, investigate and understand how the biological world was shaped by natural selection. Given the fact that the maps

and

$$
\text { genotype } \longrightarrow \text { phenotype }
$$

$$
\text { genotype } \times \text { genotype } \longrightarrow \text { genotype }
$$

(describing the sexual reproduction of diploid organisms) are extremely complicated, it is rarely possible to take a fundamental point of view and study evolution at the level of DNA. So simplifying assumptions are called for. But what course to pursue?

The approach of quantitative genetics is to simplify both the description of genotype and the two maps above. A more drastic approach is to give up on genotype and sexual reproduction completely and to concentrate on the evolution of phenotype. This forces one to pretend that reproduction is clonal. As a consequence, variation among individuals derives only from mutations (and within the framework these have to be described phenomenologically).

Models of phenotypic evolution come in (at least) two versions:

- those inspired by game theory [63, 64],

- those superimposed on (competitive) population dynamics. 
The first type focuses on behavioural traits affecting direct interaction. One specifies a trait specific pay-off for two contestants (so the pay-off also depends on the trait of the opponent) and next computes a population average over possible opponents to determine how well a type is doing, given a population composition. Next one postulates that the frequencies (i.e., the relative subpopulation sizes) change according to the relative values of these overall pay-offs or, as it is called, one assumes replicator dynamics. Reassuringly one finds a nice correspondence between the notion of ESS and asymptotic stability in the setting of replicator dynamics. See [53].

As many life history features relate to indirect interaction, via the environmental condition, there is a need for a complementary approach. As we have explained at length above, this is exactly what Adaptive Dynamics, with its focus on the ecological feedback loop, is all about.

The main achievement of Adaptive Dynamics so far is the classification of singular points for one-dimensional traits, illuminating the CSS property and the possibility of branching $[44,45,68]$. In steady progress are items like:

- $\mathrm{AD}$ as an added feature to a bifurcation diagram [20],

- multi-dimensional traits,

- when is mutation structure irrelevant for dynamics? [27, 60, 61],

- co-evolution [22],

- putting genetics underneath [21, 33, 35, 41, 50, 61, 27, 88],

- does branching lead to speciation? [21, 33, 41],

- bifurcation theory of singular points [54],

- the canonical (selection gradient) equation [7, 53, 22],

- Lotka-Volterra competition and normal forms [55].

Moreover, increasingly $\mathrm{AD}$ is used as an instrument to investigate a specific topic in evolutionary ecology, such as, e.g., the evolution of virulence in the context of hostpathogen interaction [23, 75], seed size [42] and resource use [78]. A striking feature of such investigations is that, perhaps unavoidably, it is very much a matter of choice (so, of taste) what aspects of the phenotype are considered fixed/given and which are subject to mutation and selection.

In mathematical population dynamics the notions of permanence and persistence have received a lot of attention the last 10-20 years [53]. For AD a key component is what one might call guaranteed non-persistence: give conditions on the structure of the population dynamical equations that guarantee that a successful invader outcompetes the resident in the sense of driving it to extinction (i.e., supplement the "no mutual invasibility" condition with global conditions such that together they suffice to imply trait substitution). In my opinion this is a major open problem of a relatively well-defined mathematical kind $[43,56]$.

At the biological side, a major challenge is to reconcile the tendency of ecological models to lead to competitive exclusion with the overwhelming diversity in the real world. Does evolution have a tendency to increase the number of components of $I$ and/or the complexity of the time dependence of $I$ ? Is there, perhaps, positive feedback between the complexity/dimension of the phenotype and the complexity/dimension of $I$ ? 
In these notes I have concentrated on

- concepts,

- motivation,

- classification: the catalogue,

- simple models that can be analysed by pen and paper,

and I have completely neglected

- the toolbox of numerical methods,

- ecologically relevant but rather complicated examples.

The purpose has been to provide the interested outsider, the beginner, with a starting point. If such readers are now motivated to dive into the literature, the notes have served their purpose. A list of AD papers, with some annotations, can be found at

http://users.utu.fi/evakis/ad_lit.htm

(Also see the pdf files of overheads of lectures by Kisdi and by Metz). Lots of (p)reprints and other info can be found at the web site of the IIASA AD Network:

$$
\text { www.iiasa.ac.at/Research/ADN }
$$

Acknowledgements. These notes originate from lectures given at the School on Population Dynamics, June 17-21, 2002, in Będlewo, Poland, organized by the Stefan Banach International Centre with EU support and the Summer School on Mathematical Biology, July 15-19, 2002, in Lisbon, Portugal, organized by the Centro Internacional de Matemática. It is a pleasure to thank Ryszard Rudnicki, Alessandro Margheri, Carlota Rebelo and Fabio Zanolin for the invitations and for providing the best possible stimulus by gathering an audience of young, talented and enthusiastic scientists.

Most of what I know about the subject I learned from Hans Metz, but various discussions with Geza Meszena, Kalle Parvinen and Stefan Geritz have been very inspiring as well. André de Roos and his students gave helpful feedback on an earlier version of these notes. Finally, I am most grateful to Ana Nunes for typing this manuscript.

\section{References}

[1] P. A. Abrams, Y. Harada and H. Matsuda, On the relationship between quantitative genetic and ESS models, Evolution 47 (1993), 982-985.

[2] E. Baake and W. Gabriel, Biological evolution through mutation, selection and drift: an introductory review, Ann. Rev. Comp. Phys. 7 (2002), 203-264.

[3] H. Behnke, Periodical cicades, J. Math. Biol. 40 (2000), 413-431.

[4] M. G. Bulmer, Periodical insects, Amer. Natur. 111 (1977), 1099-1117.

[5] R. Bürger, The Mathematical Theory of Selection, Recombination and Mutation, Wiley, 2000 .

[6] H. Caswell, Matrix population models, Sinauer Associates, Sunderland, Massachusetts, 2001.

[7] N. Champagnat, R. Ferrière and G. Ben Arous, The canonical equation of adaptive dynamics: a mathematical view, Selection 2 (2001), 73-84. 
[8] B. Charlesworth, Evolution in Age-Structured Populations, 2nd ed., Cambridge Univ. Press, Cambridge, 1994.

[9] E. L. Charnov, The Theory of Sex Allocation, Princeton Univ. Press, Princeton, NJ, 1982.

[10] E. L. Charnov, Life History Invariants: Some Explorations of Symmetry in Evolutionary Ecology, Oxford Univ. Press, 1993.

[11] F. B. Christiansen, Population Genetics of Multiple Loci, Wiley, 2000.

[12] D. Claessen, A. M. de Roos and L. Persson, Dwarfs and giants: cannibalism and competition in size-structured populations, Amer. Natur. 155 (2000), 219-237.

[13] J. M. Cushing, An Introduction to Structured Population Dynamics, CBMS-NSF Regional Conference Series in Applied Mathematics 71, SIAM, Philadelphia, 1998.

[14] A. M. de Roos and L. Persson, Competition in size-structured populations: mechanisms inducing cohort formation and population cycles, Theor. Pop. Biol., in press (2002).

[15] A. M. de Roos and L. Persson, Physiologically structured models - from versatile technique to ecological theory, Oikos 94 (2001), 51-71.

[16] A. M. de Roos, J. A. J. Metz, E. Evers and A. Leopoldt, A size dependent predator-prey interaction: who pursues whom?, J. Math. Biol. 28 (1990), 609-643.

[17] A. M. de Roos, A gentle introduction to physiologically structured population models, in: S. Tuljapurkar and H. Caswell (eds.), Structured-Population Models in Marine, Terrestrial, and Freshwater Systems, Chapman \& Hall, 1997, 119-204.

[18] A. M. de Roos, O. Diekmann and J. A. J. Metz, Studying the dynamics of structured population models: a versatile technique and its application to Daphnia, Amer. Natur. 139 (1992), 123-147.

[19] F. Dercole and S. Rinaldi, Evolution of cannibalistic traits: scenarios derived from adaptive dynamics, Theor. Pop. Biol. 62 (2002), 365-374.

[20] F. Dercole, R. Ferrière and S. Rinaldi, Ecological bistability and evolutionary reversals under asymmetrical competition, Evolution 56 (2002), 1081-1090.

[21] U. Dieckmann and M. Doebeli, On the origin of species by sympatric speciation, Nature 400 (1999), 354-357.

[22] U. Dieckmann and R. Law, The dynamical theory of coevolution: a derivation from stochastic ecological processes, J. Math. Biol. 34 (1996), 579-612.

[23] U. Dieckmann, J. A. J. Metz, M. W. Sabelis and K. Sigmund, Adaptive Dynamics of Infectious Diseases: in Pursuit of Virulence Management, Cambridge Studies in Adaptive Dynamics, Cambridge Univ. Press, 2002.

[24] U. Dieckmann, R. Law and J. A. J. Metz (eds.), The Geometry of Ecological Interactions, Cambridge Univ. Press, 2000.

[25] O. Diekmann, Modeling and analysing physiologically structured populations, in: V. Capasso and O. Diekmann (eds.), Mathematics Inspired by Biology, LNiM 1714, Springer, 1999.

[26] O. Diekmann and J. A. P. Heesterbeek, Mathematical Epidemiology of Infectious Diseases, Wiley, 2000.

[27] O. Diekmann, F. B. Christiansen and R. Law (eds.), Special Issue on Evolutionary Dynamics, J. Math. Biol. 34 Issues 5/6 (1996).

[28] O. Diekmann, M. Gyllenberg and J. A. J. Metz, Steady state analysis of structured population models, Theor. Pop. Biol. 63 (2003), 309-338.

[29] O. Diekmann, The many facets of evolutionary dynamics, J. Biol. Systems 5 (1997), 325339. 
[30] O. Diekmann, J. A. P. Heesterbeek and J. A. J. Metz, On the definition and the computation of the basic reproduction ratio $R_{0}$ in models for infectious diseases in heterogeneous populations, J. Math. Biol. 28 (1990), 365-382.

[31] O. Diekmann, S. D. Mylius and J. R. ten Donkelaar, Saumon à la Kaitala et Getz, sauce hollandaise, Evol. Ecol. Res. 1 261-275 (1999)

[32] O. Diekmann, M. Gyllenberg, H. Huang, M. Kirkilionis, J. A. J. Metz and H. R. Thieme, On the formulation and analysis of general deterministic structured population models. II. Nonlinear Theory, J. Math. Biology 43 (2001), 157-189.

[33] M. Doebeli and U. Dieckmann, Evolutionary branching and sympatric speciation caused by different types of ecological interactions, Amer. Natur. 156 (2000), S77-S101.

[34] B. Drossel, Biological evolution and statistical physics, arXiv: cond-mat/0101409 (2001).

[35] I. Eshel, On the changing concept of evolutionary population stability as a reflection of a changing point of view in the quantitative theory of evolution, J. Math. Biol. 34 (1996), $485-510$.

[36] I. Eshel, U. Motro and E. Sansone, Continuous stability and evolutionary convergence, J. Theor. Biol. 185 (1997), 333-343.

[37] R. Ferrière and M. Gatto, Lyapunov exponents and the mathematics of invasion in oscillatory or chaotic populations, Theor. Pop. Biol. 48 (1995), 126-171.

[38] R. Ferrière, Adaptive responses to environmental threats: evolutionary suicide, insurance, and rescue, Options, Spring 2000, IIASA; Laxenburg, Austria, 12-16 (2000).

[39] R. Ferrière and M. Gatto, Lyapunov exponents and the mathematics of invasion in oscillatory or chaotic populations, Theor.Pop. Biol. 48 (1995), 126-171.

[40] R. A. Fister, The Genetical Theory of Natural Selection, 2nd rev. ed., Dover, 1958.

[41] S. A. H. Geritz and E. Kisdi, Adaptive dynamics in diploid, sexual populations and the evolution of reproductive isolation, Proc. R. Soc. Lond. B 2670 (2000), 1671-1678.

[42] S. A. H. Geritz, E. van der Meijden and J. A. J. Metz, Evolutionary dynamics of seed size and seedling competitive ability, Theor. Pop. Biol. 55 (1999), 324-343.

[43] S. A. H. Geritz, M. Gyllenberg, F. J. A. Jacobs and K. Parvinen, Invasion dynamics and attractor inheritance, J. Math. Biol. 44 (2002), 548-560.

[44] S. A. H. Geritz, É. Kisdi, G. Meszéna and J. A. J. Metz, Evolutionarily singular strategies and the adaptive growth and branching of the evolutionary tree, Evol. Ecol. 12 (1998), $35-57$.

[45] S. A. H. Geritz, J. A. J. Metz, É. Kisdi and G. Meszéna, The dynamics of adaptation and evolutionary branching, Phys. Rev. Letters 78 (1997), 2024-2027.

[46] M. Gyllenberg and K. Parvinen, Necessary and sufficient conditions for evolutionary suicide, Bull. Math. Biol. 63 (2001), 981-993.

[47] M. Gyllenberg and J. A. J. Metz, On fitness in structured metapopulations, J. Math. Biol. 43 (2001), 545-560.

[48] M. Gyllenberg, K. Parvinen and U. Dieckmann, Evolutionary suicide and evolution of dispersal in structured metapopulations, J. Math. Biol. 45 (2002), 79-105.

[49] W. D. Hamilton, Extraordinary sex ratios, Science 156 (1967), 477-488.

[50] P. Hammerstein, Darwinian adaptation, population genetics and the streetcar theory of evolution, J. Math. Biol. 34 (1996), 511-532.

[51] M. Heino, J. A. J. Metz and V. Kaitala, Evolution of mixed maturation strategies in semelparous life histories: the crucial role of dimensionality of feedback environment, Phil. Trans. R. Soc. Lond. B 352 (1997), 1647-1655.

[52] S. Henson, Cannibalism can be beneficial even when its mean yield is less than one, Theor. Pop. Biol. 51 (1997), 109-117. 
[53] J. Hofbauer and K. Sigmund, The Theory of Evolution and Dynamical Systems, Cambridge Univ. Press, Cambridge, 1988.

[54] F. Jacobs and J. A. J. Metz, Bifurcation analysis for adaptive dynamics based on LotkaVolterra competition models, in preparation.

[55] F. J. A. Jacobs, Adaptive dynamics based on Lotka-Volterra models, in preparation.

[56] F. J. A. Jacobs, J. A. J. Metz, S. A. H. Geritz and G. Meszéna, On the mathematics of fixation in phenotypic trait evolution, in preparation.

[57] A. Kalcelnik, J. R. Krebs and C. Bernstein, The ideal free distribution and predator-prey populations, Trends Ecol. Evol. 7 (1992), 50-54.

[58] M. Kirkilionis, O. Diekmann, B. Lisser, M. Nool, A. M. de Roos, and B. Sommeijer, Numerical continuation of equilibria of physiologically structured population models. I. Theory. Mathematical Models and Methods in Applied Sciences 11 (2001), 1101-1127.

[59] S. A. L. M. Kooijman, Dynamic Energy and Mass Budgets in Biological Systems, Cambridge Univ. Press, Cambridge, 2000.

[60] O. Leimar, Multidimensional convergence stability and the canonical adaptive dynamics, in: U. Dieckmann \& J. A. J. Metz (eds.), Elements of Adaptive Dynamics, Cambridge Univ. Press, in press.

[61] C. Matessi and Di Pasquale, Long-term evolution of multilocus traits, J. Math. Biol. 34 (1996), 613-653.

[62] J. Maynard Smith and G. R. Price, The logic of animal conflict, Nature 246 (1973), 15-18.

[63] J. Maynard Smith, Evolution and the Theory of Games, Cambridge Univ. Press, 1982.

[64] M. Mesterton-Gibbons, An Introduction to Game-Theoretic Modelling, AMS, 2001.

[65] J. A. J. Metz and M. Gyllenberg, How should we define fitness in structured metapopulation models? Including an application to the calculation of evolutionarily stable dispersal strategies, Proc. Roy. Society of London B 268 (2001), 499-508.

[66] J. A. J. Metz and O. Diekmann, The Dynamics of Physiologically Structured Populations, Lecture Notes in Biomathematics 68. Springer, Berlin, 1986.

[67] J. A. J. Metz, R. M. Nisbet and S. A. H. Geritz, How should we define "fitness" for general ecological scenarios?, TREE 7 (1992), 198-202.

[68] J. A. J. Metz, S. A. H. Geritz, G. Meszéna, F. J. A. Jacobs and J. S. van Heerwaarden, Adaptive dynamics, a geometrical study of the consequences of nearly faithful reproduction, in: Stochastic and Spatial Structures of Dynamical Systems, S. J. van Strien \& S. M. Verduyn Lunel (eds.), North-Holland, Amsterdam, 1996, 183-231.

[69] J. A. J. Metz, S. Mylius and O. Diekmann, When does evolution optimise? On the relation between types of density dependence and evolutionarily stable life history parameters, Report from: IIASA, Nr.: WP-96-04 (1996).

[70] S. D. Mylius and O. Diekmann, On evolutionary stable life histories, optimization and the need to be specific about density dependence, Oikos 74 (1995), 218-284.

[71] S. D. Mylius, M. Doebeli and O. Diekmann, Can initial invasion dynamics correctly predict phenotypic substitutions?, in: Advances in Adaptive Dynamics, U. Dieckmann \& J. A. J. Metz (eds.), to appear.

[72] S. D. Mylius and O. Diekmann, The resident strikes back: Invader-induced switching of resident attractor, J. Theor. Biol. 211 (2001), 297-311.

[73] M. Nowak, An evolutionarily stable strategy may be inaccessible, J. Theor. Biol. 142 (1990), 237-241.

[74] G. A. Polis, The evolution and dynamics of intraspecific predation, Ann. Rev. Ecol. Syst. 12 (1981), 225-51. 
[75] A. Pugliese, On the evolutionary coexistence of parasites, Math. Biosc. 177/178 (2002), $355-375$.

[76] D. A. Rand, H. B. Wilson and J. M. McGlade, Dynamics and evolution: evolutionarily stable attractors, invasion exponents and phenotype dynamics, Phil. Trans. R. Soc. Lond. B 343 (1994), 261-283.

[77] D. A. Roff, The Evolution of Life Histories: Theory and Analysis, Chapman \& Hall, 1992.

[78] S. Schreiber and G. Tobiasson, The evolution of resource use, J. Math. Biol., to appear.

[79] L. A. Segel, Modeling Dynamic Phenomena in Molecular and Cellular Biology, Cambridge Univ. Press, Cambridge, 1984.

[80] H. L. Smith and P. Waltman, The Theory of the Chemostat, Cambridge Studies in Mathematical Biology 13, Cambridge Univ. Press, Cambridge, 1995.

[81] S. C. Stearns, The Evolution of Life Histories, Oxford Univ. Press, 1992.

[82] H. R. Thieme, Convergence results and a Poincaré-Bendixson trichotomy for asymptotically autonomous differential equations, J. Math. Biol. 30 (1992), 755-763.

[83] S. Tuljapurkar, An uncertain life: Demography in random environments, Theor. Pop. Biol. 35 (1989), 227-294.

[84] S. Tuljapurkar and H. Caswell, Structured-Population Models in Marine, Terrestrial, and Freshwater Systems, Population and Community Biology Series 18, Chapman \& Hall, New York, 1997.

[85] S. Tuljapurkar, Population Dynamics in Variable Environments, Springer, 1990.

[86] F. Van den Bosch, A. M. de Roos and W. Gabriel, Cannibalism as a life boat mechanism, J. Math. Biol. 26 (1988), 614-633.

[87] G. F. Webb, Theory of Nonlinear Age-Dependent Population Dynamics, Marcel Dekker, New York, 1985.

[88] F. J. Weissing, Genetic and phenotypic models of selection: is natural selection an optimizing process?, Theoretical Studies in Ecology and Evolution 18, University of Groningen, 1996.

[89] P. Yodzis, Introduction to Theoretical Ecology, Harper \& Row, 1989. 